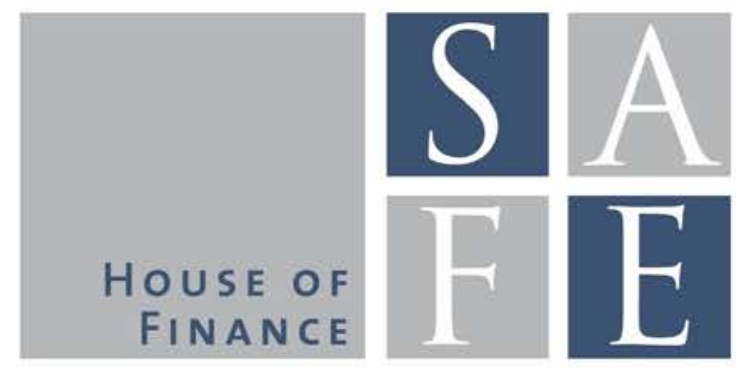

WORKING PAPER SERIES

Roberto Casarin - Michele Costola - Erdem Yenerdag

\title{
Financial Bridges and Network Communities
}

SAFE Working Paper No. 208

SAFE I Sustainable Architecture for Finance in Europe A cooperation of the Center for Financial Studies and Goethe University Frankfurt 


\section{Non-Technical Summary}

Financial crises and systemic risks proved to play central roles as shock transmitters to the real economy, threatening the stability of the economic and financial systems. These phenomena have boosted a massive interest in the literature which deeply investigated systemic risk and contagion channels on the financial systems.

Generally, systemic risk may arise as the interactions among financial institutions and markets which, consequently, lead to financial crises. A stylized fact that occurs often in real networks is the presence of a group of nodes which share common properties or play a similar role within the network. These community structures have been recognized also in finance with the presence of key nodes (community bridges) linked through short-cuts to otherwise separated communities. The case in point to better understand the role of the community structure in a network is provided by epidemiology. A parallelism with the financial stability indicates that the mitigation and prevention of the spread in infectious diseases (financial contagion) can be attained by seeking actively to immunize the super-spreaders. However, the presence of a community structure significantly affects the dynamic of the disease: immunization interventions focusing on nodes strongly linked with other communities (community bridges) are in this case more effective than the ones which aim to the highly connected nodes in the whole network. The reason is that community bridges are more relevant in spreading out contagion with respect to the nodes with fewer inter-community connections in the group: with the latter, contagion may stop before spreading out to the other communities. Hence, in a network with a community structure, classical connectedness measures can lead to the misidentification of a given SIFI at least in two cases: i) a financial institution shows a lower total degree with respect to the other nodes in the community, but a higher degree to the nodes belonging to other communities (false negative); ii) a financial institution shows a higher total degree with respect to the other nodes in the community, but a lower degree to the nodes belonging to other communities (false positive). Therefore, also in a financial network, the node immunization through the identification of highly connected nodes may not be effective in a network with community structure.

On this ground, the aim of this paper is to investigate the topology of the financial networks focusing on the detection of financial communities and community bridges to overcome the weakness of classical connectedness measure. We denote these communities as the Systemically Important Financial Communities (SIFC) defined as a group of nodes that belong to the community with the highest inter-connectivity density of the network. In this regard, we propose measures of connectedness to describe the inter- and intra community connectivity in financial networks. In the empirical analysis, we investigate the European financial system from 1996 to 2013 including all the financial firms (active and dead). Findings show a timevarying com-munity structure in the European financial networks which exhibits an increasing number of communities during periods of financial distress. By analyzing the global financial crisis and European sovereign debt crisis, our results show that the network exhibits a structure with the presence of a core block (the SIFC) acting as the shock spreader to a second block, the receiver. In both periods, insurances play a primary role in spreading shocks with respect to other financial sectors (i.e. banks). In fact, the SIFC contains a share of insurances which is more than the half of the total market and the largest financial institutions in terms of market capitalization. 


\title{
Financial Bridges and Network Communities|*
}

\author{
Roberto Casarin $\|^{\dagger} \quad$ Michele Costola $\|^{\star} \quad$ Erdem Yenerdag $\|^{\beta}$
}

This version: May 6, 2018

\begin{abstract}
The paper analyses the contagion channels of the European financial system through the stochastic block model (SBM). The model groups homogeneous connectivity patterns among the financial institutions and describes the shock transmission mechanisms of the financial networks in a compact way. We analyse the global financial crisis and European sovereign debt crisis and show that the network exhibits a strong community structure with two main blocks acting as shock spreader and receiver, respectively. Moreover, we provide evidence of the prominent role played by insurances in the spread of systemic risk in both crises. Finally, we demonstrate that policy interventions focused on institutions with inter-community linkages (community bridges) are more effective than the ones based on the classical connectedness measures and represents consequently, a better early warning indicator in predicting future financial losses.
\end{abstract}

Keywords: Systemic Risk; Financial Institutions; Network Communities; Financial Crises JEL Classification: G12; G29; C51

\footnotetext{
${ }^{*}$ We thank Ana Babus, Monica Billio, Francis Bloch, Fulvio Corsi, SangMok Lee, Gianni De Nicoló, Lorenzo Frattarolo, Mark Hallam, Loriana Pelizzon, Tuomas A. Peltonen, Brian Rogers and the participants at the Internal Macro Seminars (Washington University in St. Louis), the Domenico Econometric Workshop 2017 (Ca' Foscari University of Venice, Italy), CREDIT Conference 2017 (Ca' Foscari University of Venice, Italy), SAFE Annual Meeting 2017 (Goethe University, Frankfurt am Main, Germany), EFiC 2017 (University of Essex, Colchester, UK) and CFE 2016 Meeting (Siville, Spain). Michele Costola acknowledges financial support from the Marie Skłodowska-Curie Actions, European Union, Seventh Framework Program HORIZON 2020 under REA grant agreement n. 707070. He also gratefully acknowledges research support from the Research Center SAFE, funded by the State of Hessen initiative for research LOEWE.

${ }^{\dagger}$ Department of Economics, Ca' Foscari University of Venice, Dorsoduro 3246, 30123 Venice (Italy), r.casarin@unive.it (e-mail).

${ }^{\ddagger}$ SAFE, House of Finance, Goethe University Frankfurt, Theodor-W.-Adorno-Platz 3, 60323 Frankfurt am Main (Germany), costola@safe.uni-frankfurt.de (e-mail). (corresponding author)

${ }^{\S}$ Department of Economics, Washington University in St. Louis, One Brookings Drive Campus Box 1208, Saint Louis, MO 63130 (USA) erdemyenerdag@wustl.edu (e-mail).
} 


\section{Introduction}

Financial crises and systemic risks proved to play central roles as shock transmitters to the real economy, threatening the stability of the economic and financial systems (i.e. Allen et al., 2012, Giglio et al., 2016). These phenomena have boosted a massive interest in the literature which deeply investigated systemic risk and contagion channels on the financial systems Adrian and Brunnermeier, 2016; Barigozzi and Brownlees, 2016; Billio et al., 2012, Brownlees and Engle, 2017; Freixas et al., 2000).

Generally, systemic risk may arise as the interactions among financial institutions and markets which, consequently, lead to financial crises (Allen and Carletti, 2013). The analysis of financial linkages is a powerful tool in monitoring the structure of the financial system to pursuit financial stability (Diebold and Yilmaz, 2015; Scott, 2016). For example, the Financial Stability Board (2009) considers interconnectedness as a key criteria to identify the systemic importance of the financial institutions for the financial system. Highly interconnected financial networks can be "robust-yet-fragile": connectedness acts as a shock-absorber for a given range, while beyond that range, it becomes a "shock-propagator mechanism" where robustness turns to fragility generating systemic risks (Haldane, 2013). Indeed, the characteristics that make a financial network more resilient are the same that under different conditions bring instability on the financial system (Acemoglu et al., 2015). To enhance the resilience of the financial system and mitigate systemic risk, policy makers aim to identify the systemically important financial institutions (SIFIs) (Freixas et al., 2000; Thomson, 2010). Consequently, with the studies of topologies and connectedness of the financial networks, new different indicators have been suggested to authorities to measure systemic risk (e.g., see Billio et al., 2012; Diebold and Yllmaz, 2014). For instance, the study of global measures (e.g. density and assortativity) are used to describe the structure of the financial networks and hence the fragility of the systems while the use of local measures (i.e. degree and centrality) at the node level allow to detect the systemically important institutions (Billio et al., 2017; Hurd, 2016, e.g.). A stylized fact that occurs often in networks is that the probability of having an edge between a pair of vertices that is not equal across all possible pairs. Differently from random graphs, real networks tends to display inhomogeneity not only globally, but also locally with high concentration of edges within groups of nodes and low concentrations between groups. This phenomenon is known as community structure or network modularity (Leicht and Newman, 2008, Newman, 2010, 2006). In general terms, a community is a group of nodes which share common properties or play a similar role within the network 
(Fortunato, 2010). Community structures in real networks have been recognized also in finance with the presence of key nodes linked through short-cuts to otherwise separated communities (Haldane, 2013). These key-nodes act as community bridges. Loepfe et al. (2013) show that in case of large shocks in financial networks, modularity acts as a trigger on the transition mechanism switching from safe to risky regime.

Community structures have not been fully investigated in financial literature. Notable exceptions are represented by De Souza et al. (2016), Puliga et al. (2016) and Bargigli and Gallegati (2013). Bargigli and Gallegati (2013) investigate credit communities on a Japanese bank-firm weighted and directed bipartite network finding a strengthening of Japanese communities over time. Puliga et al. (2016) find through an accounting network that regional bank communities change weakening geographically boundaries while De Souza et al. (2016) identify Brazilian banking communities through inter-banking exposures and find that a large part of them includes non-large banks.

The case in point to better understand the role of the community structure in a network is provided by epidemiology. A parallelism with the financial stability indicates that the mitigation and prevention of the spread in infectious diseases (financial contagion) can be attained by seeking actively to immunize the super-spreaders (Haldane and May, 2011). However, the presence of a community structure significantly affects the dynamic of the disease: immunization interventions focusing on nodes strongly linked with other communities (community bridges) are in this case more effective than the ones which aim to the highly connected nodes in the whole network (Salathé and Jones, 2010). The reason is that community bridges are more relevant in spreading out contagion with respect to the nodes with fewer inter-community connections in the group: with the latter, contagion may stop before spreading out to the other communities. More generally, a critical aspect of node centrality measures is their sensitivity to the degree heterogeneity. In presence of strong degree heterogeneity, e.g. hubs and core-periphery structures, most of the components have null centrality. Hence, in a network with a community structure, classical connectedness measures can lead to the misidentification of a given SIFI at least in two cases: i) a financial institution shows a lower total degree with respect to the other nodes in the community, but a higher degree to the nodes belonging to other communities (false negative); ii) a financial institution shows a higher total degree with respect to the other nodes in the community, but a lower degree to the nodes belonging to other communities (false positive). Therefore, also in a financial network, the node immunization through the identification of highly connected nodes may not be effective in a network with community structure (Karrer 
and Newman, 2011).

On this ground, the aim of this paper is to investigate the topology of the financial networks focusing on the detection of financial communities and community bridges to overcome the weakness of classical connectedness measure. We denote these communities as the Systemically Important Financial Communities (SIFC) defined as a group of nodes that belong to the community with the highest inter-connectivity density of the network. In this regard, we propose measures of connectedness to describe the inter- and intra community connectivity in financial networks.

Within the scope of Stochastic Block Models, we identify the communities based on the stochastic equivalence principle in generating the network structure. We rely on the Weighted Stochastic Block Model (WSBM) which considers both the edge existence and the edge weight of the network (Aicher et al., 2014). The WSBM allows to have a compact characterization of the network structure through the blocks and represents a generalization of the Stochastic Block model (SBM) introduced by (Holland et al., 1983). Hałaj and Kok (2013) show that SBM can be used successfully to simulate contagion networks and cascades, since they allow to circumvent the bias problem in standard random network models which tend to underestimate tails and contagion risk. To our knowledge, this paper is the first to introduce this approach in the financial economics literature.

In the empirical analysis, we investigate the European financial system from 1996 to 2013 including all the financial firms (active and dead). Dynamic networks of European financial institutions are generated through the Granger-causality using a rolling window estimation as performed in Billio et al. (2012). This bivariate approach for network estimation allows to deal easily with large datasets and delisting in stocks markets, and therefore to avoid the survivorship bias (Shumway, 1997). Clearly, the WSBM approach is independent by the chosen methodology in the network estimation and alternative techniques such as graph-based approaches (i.e. Ahelegbey et al., 2016a) and sparse models (i.e. Ahelegbey et al., 2016c; Hautsch et al., 2015) can be applied.

Findings show a time-varying community structure in the European financial networks which exhibits an increasing number of communities during periods of financial distress. We consider the strengthening of the community structure as an early warning signal of network disintegration given the rise of heterogeneous connectivity patterns which lead to a different dynamic of shocks affecting financial institutions. Moreover, using the proposed community connectivity measures, we find that the density of the financial network is mainly driven by the inter-linkages 
across the communities confirming the primary role of financial bridges in the spread of shocks across the communities and consequently, to the entire network. Consequently, inter connectedness represents a better early warning indicator in terms of future financial losses with respect to the classical connectedness measures. By analysing the global financial crisis and European sovereign debt crisis, our results show that the network exhibits a structure with the presence of a core block (the SIFC) acting as the shock spreader to a second block, the receiver. In both periods, insurances play a primary role in spreading shocks with respect to other financial sectors (i.e. banks). In fact, the SIFC contains a share of insurances which is more than the half of the total market and the largest financial institutions in terms of market capitalization. Differently from Billio et al. (2012) which provide evidence of interconnectedness among insurers and other financial institutions, we show that insurances not only are part of the game but also play a prominent role in the spread of systemic risk. Finally, we perform an immunization exercise during the European sovereign debt crisis showing that removing the institutions with highest inter community degrees is more effective then the ones with the highest community inter out degrees.

The remainder of the paper is organized as follows. Section 2 presents the SBMs and inference. Section 3 introduces the dynamic network extraction and the community connectivity measures. Section 4 presents the empirical analysis on the European financial system while Section 5.2 the Financial bridges as early warning indicator and the policy implications. Finally, Section 6 concludes.

\section{Modelling Communities}

In this section, we present time-varying Stochastic Block Model (SBM) and inference for community detection which consider both edge and weight information.

\subsection{Stochastic Block Models}

We extend to a dynamic context the SBM and Weighted Stochastic Block Model (WSBM) models given in Aicher et al. (2014).

Let $G_{t}=\left(V_{t}, E_{t}\right), t=1, \ldots, T$ be a time sequence of networks, with vertex set $V_{t}$ and edge set $E_{t}$, and let $A_{t}$ be the $n_{t} \times n_{t}$ adjacency matrix of the network $G_{t}$, which contains binary values representing edge existence, i.e. $A_{i j, t} \in\{0,1\}$ with $A_{i j, t}=1$ if $(i, j) \in E_{t}$ and $A_{i j, t}=0$ otherwise, $i, j=1, \ldots, n_{t}$ where $n_{t}$ is the cardinality of $V_{t}$. See Elliott et al. (2014). 
We assume $t_{0}<t_{1}<t_{2}<\ldots<t_{M}<t_{M+1}$, with $t_{0}=1$ and $t_{M+1}=T$, is a sequence of change points. The network parameters and the label vector are constant within a sub-period, but change over sub-periods $\left[t_{m-1}, t_{m}\right]$, and the edge distribution for the network at time $t$ is

$$
g\left(A_{t} \mid \mathbf{z}, \boldsymbol{\theta}\right)= \begin{cases}f\left(A_{t} \mid \mathbf{z}_{1}, \boldsymbol{\theta}_{1}\right) & 0<t \leq t_{1} \\ f\left(A_{t} \mid \mathbf{z}_{2}, \boldsymbol{\theta}_{2}\right) & t_{1}<t \leq t_{2} \\ \cdots & \ldots \\ f\left(A_{t} \mid \mathbf{z}_{M}, \boldsymbol{\theta}_{M}\right) & t_{M}<t \leq T\end{cases}
$$

where $f\left(A \mid \mathbf{z}_{M}, \boldsymbol{\theta}_{M}\right)$ is an edge probability model discussed in the following, $\boldsymbol{\theta}=\left(\boldsymbol{\theta}_{1}, \ldots, \boldsymbol{\theta}_{M}\right)$ is the collection of period-specific parameters and $\mathbf{z}=\left(\mathbf{z}_{1}, \ldots, \mathbf{z}_{M}\right)$ is the collection of periodspecific latent variable vectors.

In the following we present the models for the edge probabilities and drop for the sake of simplicity the time subscript $t$. In the basic SBM, there are $K$ latent groups of nodes and the probability $p_{i j}$ of an edge between nodes $i$ and $j$, i.e. $\operatorname{Pr}((i, j) \in E)$ depends on the groups the two nodes belong, that is

$$
p_{i j}=\theta_{z_{i} z_{j}}^{A_{i j}}\left(1-\theta_{z_{i} z_{j}}\right)^{1-A_{i j}}
$$

$i, j=1, \ldots, n$, where $z_{i} \in\{1, \ldots, K\}$ indicates the which group the node $i$ belongs to. The existence probability of an edge $A_{i j}$ is given by the parameter $\theta_{z_{i}, z_{j}}$ that depends only on the membership of nodes $i$ and $j$. Note that, in Equation 1 the $A_{i j}$ s are conditionally independent given $z_{i}, i=1, \ldots, n$ and $\theta_{k l}, k, l=1, \ldots, K$. The probability that $z_{i}=k$ is equal to $\gamma_{i k}$, with $k=1, \ldots, K$. We assume $\gamma_{i k}$ equal to $\gamma_{k}$. The number of latent groups, $K$, is a free parameter that must be chosen before the model and it controls the model's complexity.

Let $\mathbf{z}=\left(z_{1}, \ldots, z_{n}\right)$ be the $(n \times 1)$ vector that contains the labels of the nodes. Then, vector $z$ represents the partition of the nodes into $K$ blocks and each pair of groups $(k, l)$ represents a bundle of edge between the groups. The parameter $\boldsymbol{\theta}$ in Equation 1 represents a $(K \times K)$ matrix, the affinity matrix, with $(l, k)$-element the edge probability parameter $\theta_{l k}$. From Equation 1 the joint probability distribution of the edge existence for a given network $G$ can be written as follows:

$$
f(A \mid \mathbf{z}, \boldsymbol{\theta})=\prod_{(i, j) \in E} \exp \left(A_{i j} \cdot \log \left(\frac{\theta_{z_{i} z_{j}}}{1-\theta_{z_{i} z_{j}}}\right)+\log \left(1-\theta_{z_{i} z_{j}}\right)\right)
$$


which belongs to the exponential family, since it has kernel

$$
f(A \mid \mathbf{z}, \boldsymbol{\theta}) \propto \exp \left(\sum_{(i, j) \in E} \boldsymbol{\tau}\left(A_{i j}\right) \cdot \boldsymbol{\eta}\left(\theta_{z_{i} z_{j}}\right)\right)
$$

where $\boldsymbol{\tau}(x)=(x, 1)^{\prime}$ is the vector-valued function of sufficient statistics of a Bernoulli distribution and $\boldsymbol{\eta}(x)=(\log (x /(1-x)), \log (1-x))$ a vector-valued function of natural parameters. This is a basic and classical SBM for unweighted networks since, as they are defined, the functions $\tau$ and $\boldsymbol{\eta}$ produce binary edge values.

The latent allocation variables $z_{i}$ follow a discrete uniform distribution

$$
z_{i} \sim \mathcal{U}_{\{1, \ldots, K\}}
$$

i.i.d. for $i=1, \ldots, n$ which implies $\gamma_{k}=1 / K$. For the edge probability parameter $\theta$ we assume conjugate non-informative prior, that is

$$
\theta_{k k^{\prime}} \sim \mathcal{U}_{[0,1]}
$$

i.i.d. for $k, k^{\prime}=1, \ldots, K$.

With a different and appropriate choice of the functions $\boldsymbol{\tau}$ and $\boldsymbol{\eta}$ a WSBM can be established by weights that are drawn from an exponential family distribution over the domain of $\boldsymbol{\tau}$. In this case, each $\theta_{z_{i} z_{j}}$ denotes the parameters governing the weight distribution of the edge bundle $\left(z_{i} z_{j}\right)$ and the edge weight probability is

$$
f(A \mid \mathbf{z}, \boldsymbol{\theta}) \propto \exp \left(\sum_{(i, j) \in W} \boldsymbol{\tau}\left(A_{i j}\right) \cdot \boldsymbol{\eta}\left(\theta_{z_{i} z_{j}}^{(w)}\right)\right)
$$

where the pair of functions $(\boldsymbol{\tau}, \boldsymbol{\eta})$ for the real valued edge weights is needed. Networks with real valued edge weights can be modelled by an exponential family distribution. We consider a normal distribution with parameters $\mu$ and $\sigma^{2}$ and define with $\boldsymbol{\tau}(x)=\left(x, x^{2}, 1\right)$ the vector of sufficient statistics and with $\boldsymbol{\eta}(\boldsymbol{\theta})=\left(\mu / \sigma^{2},-1 /\left(2 \sigma^{2}\right),-\mu^{2} /\left(2 \sigma^{2}\right)\right)$ the natural parameter vector.

The latent allocation variables $z_{i}$ follow a discrete uniform distribution that is

$$
z_{i} \sim \mathcal{U}_{\{1, \ldots, K\}}
$$


i.i.d. for $i=1, \ldots, n$. For the parameter $\boldsymbol{\theta}$ we assume conjugate non-informative prior, that is

$$
\sigma^{2} \sim \frac{1}{\sigma^{2}} \mathbb{I}_{\mathbb{R}^{+}}\left(\sigma^{2}\right), \quad \mu \sim \mathbb{I}_{\mathbb{R}}(\mu)
$$

The SBM and pure WSBM models produce complete graphs which can be an undesirable features in large dimension networks.In order to model sparse networks by SBM and WSBM, Aicher et al. (2014) assumes $A_{i j}=0$ as a directed edge from node $i$ to $j$ is existed with zero weight, thus parse networks can be modelled with two types of information, edge existence and edge weight values, in together by a simple tuning parameter, that is:

$$
f(A \mid \mathbf{z}, \boldsymbol{\theta}) \propto \exp \left(\alpha \sum_{(i, j) \in E} \boldsymbol{\tau}_{e}\left(A_{i j}\right) \cdot \boldsymbol{\eta}_{e}\left(\theta_{z_{i} z_{j}}^{(e)}\right)+(1-\alpha) \sum_{(i, j) \in W} \boldsymbol{\tau}_{w}\left(A_{i j}\right) \cdot \boldsymbol{\eta}_{e}\left(\theta_{z_{i} z_{j}}^{(w)}\right)\right)
$$

where the pair $\left(\boldsymbol{\tau}_{e}, \boldsymbol{\eta}_{e}\right)$ denotes the family of edge existence distribution and the pair $\left(\boldsymbol{\tau}_{w}, \boldsymbol{\eta}_{w}\right)$ denotes the family of edge-weight distribution, $\alpha \in[0,1]$ is a simple tuning parameter that combines their contributions in the edge probability. $E$ is the set of observed interactions (including non-edges) and $W$ is the set of weighted edges with $W \subset E$.

If $\alpha=1$ in Equation 8 then the model reduces to SBM in Equation 1, and if $\alpha=0$ the model ignores edge existence information then we call such models as pure WSBM (pWSBM). When $0<\alpha<1$, the edge distribution combines both information set, and if $\alpha=0.5$ the model is called balanced WSBM (Aicher et al., 2014).

In Equation 8 for the SBM part we assume $\boldsymbol{\tau}_{e}(x)=(x, 1)^{\prime}$ and $\boldsymbol{\eta}_{e}(x)=(\log (x /(1-x)), \log (1-$ $x)$ ). For the pure WSBM part in Equation 8 we assume $\boldsymbol{\tau}_{w}(x)=\left(x, x^{2}, 1\right)$ and $\boldsymbol{\eta}_{w}(\boldsymbol{\theta})=$ $\left(\mu / \sigma^{2},-1 /\left(2 \sigma^{2}\right),-\mu^{2} /\left(2 \sigma^{2}\right)\right)$ the natural parameter vector. Finally we define with $\boldsymbol{\theta}=\{P, \boldsymbol{\mu}, \boldsymbol{\sigma}\}$ the parameter vector with $P=\left(\theta_{i j}\right)_{i j}, \boldsymbol{\mu}=\left(\mu_{1}, \ldots, \mu_{K}\right)$ and $\boldsymbol{\sigma}=\left(\sigma_{1}, \ldots, \sigma_{K}\right)$.

\subsection{Bayesian Inference for Stochastic Block Models}

Let $A_{m-1: m}$ be the collection of matrices from time $t_{m-1}+1$ to time $t_{m}$, i.e. $A_{m-1: m}=$ $\left\{A_{t_{m-1}+1}, \ldots, A_{t_{m}}\right\}$. In our change-point models, we assume in each sub-sample the allocation and parameter vectors, $\mathbf{z}_{m}$ and $\boldsymbol{\theta}_{m}$, respectively, are constant, thus the likelihood function of our SBM is

$$
\mathcal{L}\left(A_{1: M} \mid \mathbf{z}_{1: M}, \boldsymbol{\theta}_{1: M}\right) \propto \prod_{m=1}^{M} \exp \left(\sum_{i j} \boldsymbol{\tau}_{e}\left(\bar{A}_{i j, m}\right) \cdot \boldsymbol{\eta}_{e}\left(\theta_{k, z_{i m} z_{j m}}\right)\right)
$$


where $\bar{A}_{i j, m}$ is the $(i, j)$-the element of the matrix

$$
\bar{A}_{m}=\sum_{t=t_{m-1}+1}^{t_{m}} A_{t}
$$

and $\boldsymbol{\tau}_{e}(x)=(x, d)$ is the vector-valued function of sufficient statistics and $\boldsymbol{\eta}_{e}(\theta)=(\log (\theta /(1-$ $\theta)), \log (1-\theta))$ is the vector-valued function of natural parameters. See Appendix A for a proof.

Since for the WSBM each edge bundle $\left(z_{i m} z_{j m}\right)$ is now parametrized by a mean and variance, $\theta_{z_{i m} z_{j m}}=\left(\mu_{z_{i m} z_{j m}}, \sigma_{z_{i m}, z_{j m}}^{2}\right)$, the likelihood of the pure WSBM can be written as:

$$
\mathcal{L}\left(A_{1: M} \mid \mathbf{z}_{1: M}, \boldsymbol{\theta}_{1: M}\right) \propto \prod_{m=1}^{M} \exp \left(\sum_{i j} \boldsymbol{\tau}_{w}\left(\bar{A}_{i j, m}\right) \cdot \boldsymbol{\eta}_{w}\left(\theta_{k, z_{i m} z_{j m}}\right)\right)
$$

where $\boldsymbol{\tau}_{w}(x)=\left(x, x^{2}, d\right)$ and $\boldsymbol{\eta}_{w}(\boldsymbol{\theta})=\left(\mu / \sigma^{2},-1 /\left(2 \sigma^{2}\right),-\mu^{2} /\left(2 \sigma^{2}\right)\right)$. See Appendix A for a proof.

The likelihood of the general WSBM can be obtained by combining Equation 9 and Equation 11 with combining parameter $\alpha \in[0,1]$, that is.

$$
\begin{aligned}
\mathcal{L}\left(A_{1: M} \mid \mathbf{z}_{1: M}, \boldsymbol{\theta}_{1: M}\right) & =\exp \left(\alpha\left(\sum_{m=1}^{M} \sum_{i j} \boldsymbol{\tau}_{e}\left(\bar{A}_{i j, m}\right) \cdot \boldsymbol{\eta}_{e}\left(\theta_{m, z_{i m} z_{j m}}\right)\right)\right. \\
& \left.+(1-\alpha)\left(\sum_{m=1}^{M} \sum_{i j} \boldsymbol{\tau}_{w}\left(\bar{A}_{i j, m}\right) \cdot \boldsymbol{\eta}_{w}\left(\theta_{m, z_{i m} z_{j m}}\right)\right)\right)
\end{aligned}
$$

where the pair $\left(\boldsymbol{\tau}_{e}, \boldsymbol{\eta}_{e}\right)$ denotes the family of edge existence distribution and the pair $\left(\boldsymbol{\tau}_{w}, \boldsymbol{\eta}_{w}\right)$ denotes the family of edge-weight distribution. $E$ is the set of observed interactions (including non-edges) and $W$ is the set of weighted edges with $W \subset E$ and $\alpha$ is a tuning parameter which combines the contributions of edge existence and edge weight information in the likelihood function. See Appendix A for a proof.

The posterior distribution of model in Equation 8 is intractable, but can be approximated by using several methods. Markov Chain Monte Carlo (MCMC) can be an efficient approach with a high computational cost. Thus, in this paper, we follow Aicher et al. (2014) and apply variational Bayes methods to circumvent this issue. Variational Bayes approximation has been successfully applied in many fields such as neural science (e.g. Penny et al., 2003), and biostatistics (e.g. Teschendorff et al., 2005) where high dimensional models or large datasets make MCMC methods not feasible. Variational Bayes approximation is now popular also in statistics (e.g., see McGrory 
and Titterington (2007), Titterington (2004), Wand et al. (2011), Pham et al. (2013), Huang et al. (2013)). See also Blei et al. (2017) for an up-to-date review.

The parameters $K$, number of blocks, and $\alpha$, tuning parameter, are crucial in our application.

Selection of optimal $K$ and $\alpha$ can be achieved by applying Bayes factor. The selection of the tuning parameter, $\alpha$, is left and we considered in the application three different values of $\alpha$ : 0 (pure WSBM), 0.5 (balanced WSBM) and 1 (basic SBM).

\section{Dynamic Networks}

The relations of institutions in the financial system can be characterized very efficiently by networks with nodes, as financial institutions, and edges, as financial relationships (e.g., see Franklin Allen, 2009). The analysis of the linkages to the edges of financial networks can be carried out studying topological features of the networks. In this section, we present our new community connectivity measures.

\subsection{Community Connectivity Measures}

As in the previous section let $z_{i t} \in\left\{1, \ldots, K_{t}\right\}$ be the allocation variable indicating the community to which the institution $i$ belongs to at time $t, K_{t}$ the number of communities, $n_{k t}$ the number of institutions in the $k$-th community, and $n_{t}$ the number of institutions in the network at time $t$.

The in- and out-degree of a node $i$ that are

$$
d_{i t}^{+}=\sum_{j=1}^{n_{t}} A_{j i, t}, \quad d_{i t}^{-}=\sum_{j=1}^{n_{t}} A_{i j, t}
$$

respectively, can be decomposed as

$$
\begin{aligned}
& d_{i t}^{+}=d_{i t}^{+, I N T R A}+d_{i t}^{+, I N T E R}, \\
& d_{i t}^{-}=d_{i t}^{-, I N T R A}+d_{i t}^{-, I N T E R},
\end{aligned}
$$

where the intra- and inter-community degrees

$$
\begin{aligned}
& d_{i t}^{+, I N T R A}=\sum_{j=1}^{n_{t}} A_{j i, t} \mathbb{I}\left(z_{i t}=z_{j t}\right), \quad d_{i t}^{+, I N T E R}=\sum_{j=1}^{n_{t}} A_{j i, t}\left(1-\mathbb{I}\left(z_{i t}=z_{j t}\right)\right), \\
& d_{i t}^{-, I N T R A}=\sum_{j=1}^{n_{t}} A_{i j, t} \mathbb{I}\left(z_{i t}=z_{j t}\right), \quad d_{i t}^{-, I N T E R}=\sum_{j=1}^{n_{t}} A_{i j, t}\left(1-\mathbb{I}\left(z_{i t}=z_{j t}\right)\right),
\end{aligned}
$$


measure the connectivity of the node (institution) $i$ with the other nodes (institutions) in the same community (INTRA) and with the nodes of the other communities (INTER). The out (in) inter-linkages allows to identify which nodes play the role of financial bridges as shock spreaders (receivers) to (from) other communities. In particular, the inter-linkages among a given community to another one can be viewed as a sub-bipartite network. Appendix B shows the type of potential connectivity patterns among the the communities.

Hence, the density of the network,

$$
f_{t}=\frac{1}{2 n_{t}\left(n_{t}-1\right)} \sum_{i=1}^{n_{t}}\left(d_{i t}^{+}+d_{i t}^{-}\right)
$$

can be represented as the convex combination

$$
f_{t}=w_{1 t} f_{t}^{I N T R A}+w_{2 t} f_{t}^{I N T E R},
$$

of intra- and inter-community densities

$$
f_{t}^{I N T R A}=\frac{1}{2 c_{1 t}} \sum_{i=1}^{n_{t}}\left(d_{i t}^{+, I N T R A}+d_{i t}^{-, I N T R A}\right), \quad f_{t}^{I N T E R}=\frac{1}{2 c_{2 t}} \sum_{i=1}^{n_{t}}\left(d_{i t}^{+, I N T E R}+d_{i t}^{-, I N T E R}\right)
$$

where $f_{t}^{I N T R A}$ is equal to 1 if all the community sub-networks are complete graphs and $f_{t}^{I N T E R}$ is equal to 1 if all the inter-community sub-networks are complete graphs. If there is no edges between the communities, i.e. $f_{t}^{I N T E R}=0$, then there is no risk of spreading contagion from one community to another. In this case, each community can be viewed as a separated network and the identification of the SIFIs reduces to find central institutions within each community as in standard connectedness measures (Billio et al., 2012; Diebold and Yllmaz, 2014; Diebold and Yilmaz, 2015). The normalizing constants

$$
c_{1 t}=\sum_{k=1}^{K_{t}} n_{k t}\left(n_{k t}-1\right), \quad c_{2 t}=\sum_{k=1}^{K_{t}} \sum_{l \neq k} n_{k t} n_{l t}
$$

provide the total degree of the sub-networks when the corresponding graphs are complete. The weights in the convex combination

$$
w_{1 t}=\frac{1}{n_{t}\left(n_{t}-1\right)} c_{1 t}, \quad w_{2 t}=\frac{1}{n_{t}\left(n_{t}-1\right)} c_{2 t}
$$



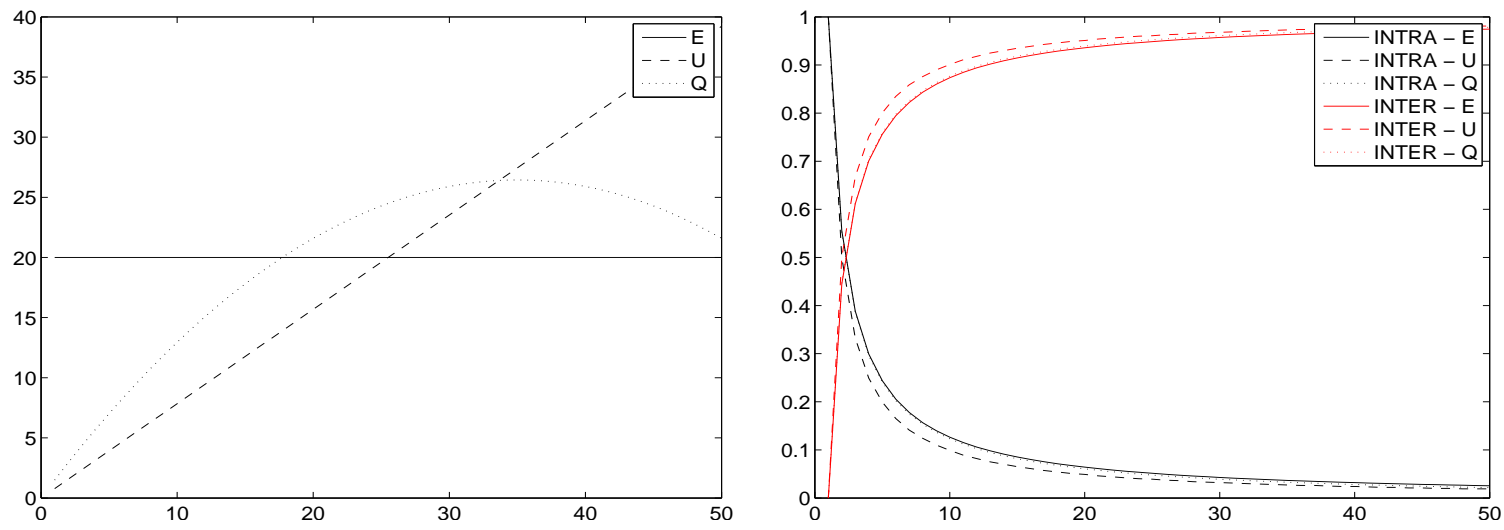

Figure 1: Number of nodes $n_{k t}$ (left plot, vertical axis) and intra- and inter-comunity densities (right plot, vertical axis) per community $k=1, \ldots, 50$ (horizontal axis), assuming a Dirac's distribution, $n_{k t} \propto n_{t} / K_{t}$ (solid lines), a uniform distribution, $n_{k t} \propto n_{t} k$ (dashed lines) and translated symmetric beta distribution, $n_{k t} \propto n_{t}(70-k) k$ (dotted lines).

are functions of the number of nodes in each community and satisfy $w_{1 t}+w_{2 t}=1, \forall t$.

Increasing the number of communities $K_{t}$ the importance of the inter-community density increases (see red line in the right plot of Figure 1). For $K_{t}>2$ the inter-community density is more relevant than the intra-community in the whole connectivity of the network. Also, we study the effect of the distribution of the community sizes on the contribution of the inter-community density to the network density and find that (see left plot of Figure 1):

- a Dirac distribution, corresponds to the case of communities of equal size (solid);

- a uniform distribution, represents the case all community sizes are equally reppresented in the network (dashed);

- a symmetric beta distributed community size,implies a community with a large size and then various communities with smaller size (dotted).

Comparing the different lines (solid, dashed and dotted) in the right plot of Fig. 1 one can see that for $K_{t}>2$ the largest weight for the inter-community density is associated to the uniform distribution.

\section{Empirical Analysis}

In this section, we analyse of European financial market and its community structure during the period of 1996-2013 considering the balanced WSBM with $\alpha=0.5$. Further details on the estimation of the optimal number of blocks and the model selection according $\alpha$ are reported 
in Appendix C. Finally, we discuss the community structure of the network on three specific moments: the Early 2000s recession, the 2007-2008 global financial crisis and the 2010-2013 European sovereign debt crisis.

\subsection{Data Description}

The dataset is composed by the daily closing price series at a daily frequency from $29^{\text {th }}$ December 1995 to $16^{\text {th }}$ January 2013 of all the European financial institutions active and dead in order to cope with survivorship bias (Shumway, 1997). Financial institutions are classified under the Industry Classification Benchmark (ICB) which includes four levels of industry classification for each company. The ICB code class 8000 represents the Financials Industry. In our analysis, we consider the four super sectors (second level) of the Financial Industry: 11 i) Banks (code 8300); ii) Insurance (code 8500); iii) Real Estate (code 8600) and iv) Financial Services (code 8700). Data have been downloaded from Datastream ${ }^{\circledR}$ using Thomson Reuters Worldscope lists ${ }^{\circledR}$. The built database is suitable to represents the European financial systems since it covers a total of 770 European financial firms $\mathrm{s}^{2}$ which are traded in 10 European financial markets (core and peripheral). The markets with the number of institutions in round brackets are: Austria (15), Belgium (30), France (81), Germany (136), Greece (56), Ireland (5), Italy (95), Netherlands (4), Spain (40) and United Kingdom (304).

The pairwise Granger causalities are estimated on daily returns using a rolling window approach with a length for each window of 252 observations (approximately 1 year). We obtain a total of 4197 adjacency matrices, $A_{t}$, during the period from $17^{\text {th }}$ December 1996 to $16^{\text {th }}$ January $20133^{3}$ We estimate dynamic networks of European financial institutions through pairwise Grangercausality as in Billio et al. (2012) and then implement our WSBMs. See also Ahelegbey et al. (2016ab); Hautsch et al. (2015) for alternative network extraction methods.

\subsection{Community Structure and Financial Bridges}

In the SBM framework, the presence of a community structure indicates that there are group of nodes with different level of connectivity. It is worth noting that the definition of community is more general with respect to the usual assortativity based definition (see Aicher et al., 2014).

\footnotetext{
${ }^{1}$ Further details can be found at ICB's website: www.icbenchmark.com In particular, it is available the Industry Classification Benchmark document with Structure and Definitions for the type of Industry (Financials Industry at Section 7.9) at http://www.ftse.com/products/downloads/ICB_Rules.pdf.

${ }^{2}$ The complete list is available upon request to the authors.

${ }^{3}$ The estimations have been parallelized and implemented in Matlab on the SCSCF (Sistema di Calcolo Scientifico Ca' Foscari) cluster multiprocessor system which consists of 4 nodes; each comprises four Xeon E5-4610 v2 2.3GHz CPUs, with 8 cores, 256GB ECC PC3-12800R RAM, Ethernet 10Gbit, 20TB hard disk system with Linux.
} 
In a network with no community structure (one community), all the financial institutions show an homogeneous connectivity level and thus, the spread of shocks will affects the whole system in the same manner. Clearly, this does not imply the absence of systemic risk. A network could be highly interconnected showing the same patterns of connectivity between the nodes where the robust-yet-fragile property is preserved leading to a resilient network for a given range of shocks but unstable above that range. Conversely, a network with community structure means the presence of an heterogeneous system composed by homogeneous groups with different connectivity patterns. Consequently, a strengthening in the structure get to a disintegration of the financial network in many sub-networks where each block of institutions may play a different role in terms of financial connectedness within and without the group. These patterns define the type of structure in terms of assortativity/disassortivity (if linkages are mainly within or without the group, respectively) and/or core-periphery (the presence of a core community).

As a first point, we are interested on the analysis of community structure of the European financial network in the period. The community structure is given by the series of the optimal block numbers, $K$, as shown in Figure 2 along with the CISS (Hollo et al. 2012). The CISS is a

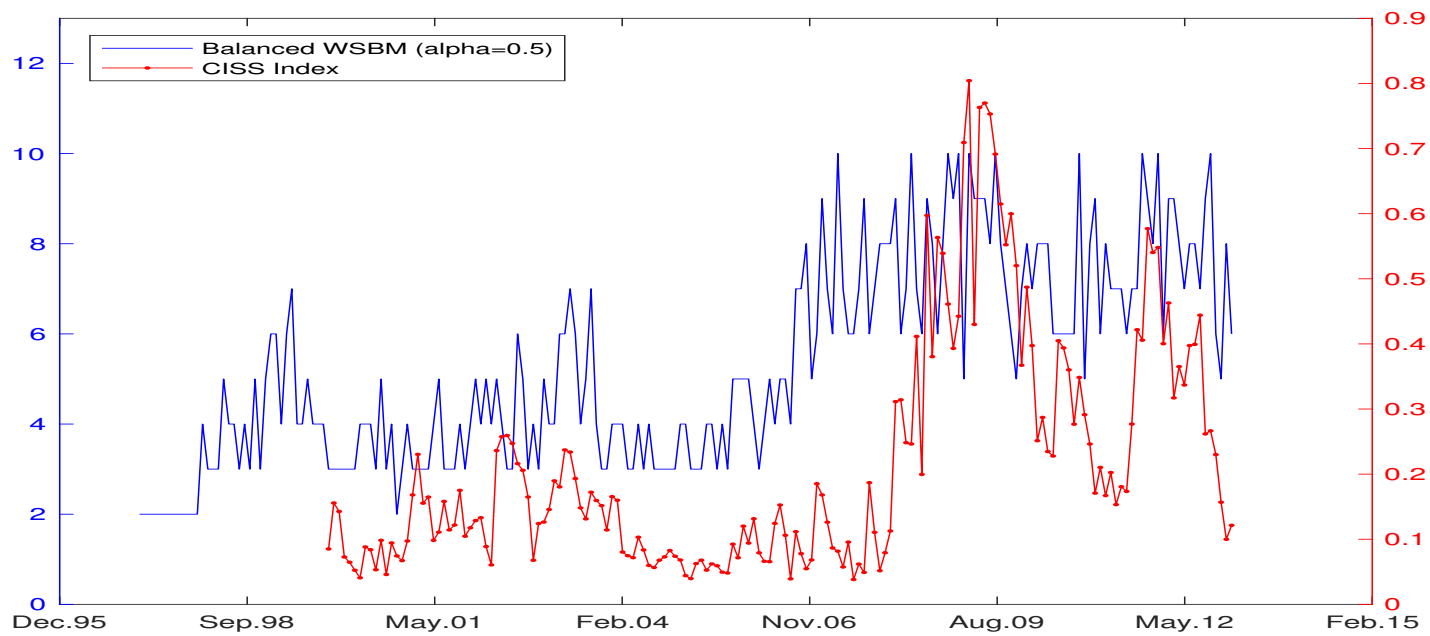

Figure 2: The number of communities $K$ (black solid line) and the CISS indicator (red dashed line) by Hollo et al. (2012) during 1996-2013.

composite indicator of contemporaneous stress in the financial system released periodically by the European Central Bank (ECB). Even if it is not sophisticated to describe the dynamic of the financial system, as any classical financial stress indicators, it is useful to monitor the current level of distress in the system. In this regard, the two series exhibit a significant and positive Spearman's correlation equal to 0.6098 which suggests that during financial stress periods a strengthening of the structure is likely to be expected. As robustness checks, we include in 
Appendix D the estimation of the optimal block numbers with different window lengths. During financial stress periods, the granularity of the community structure increases as shown in Figure 3 which reports the size of the communities as the total percentage of the network during the period.

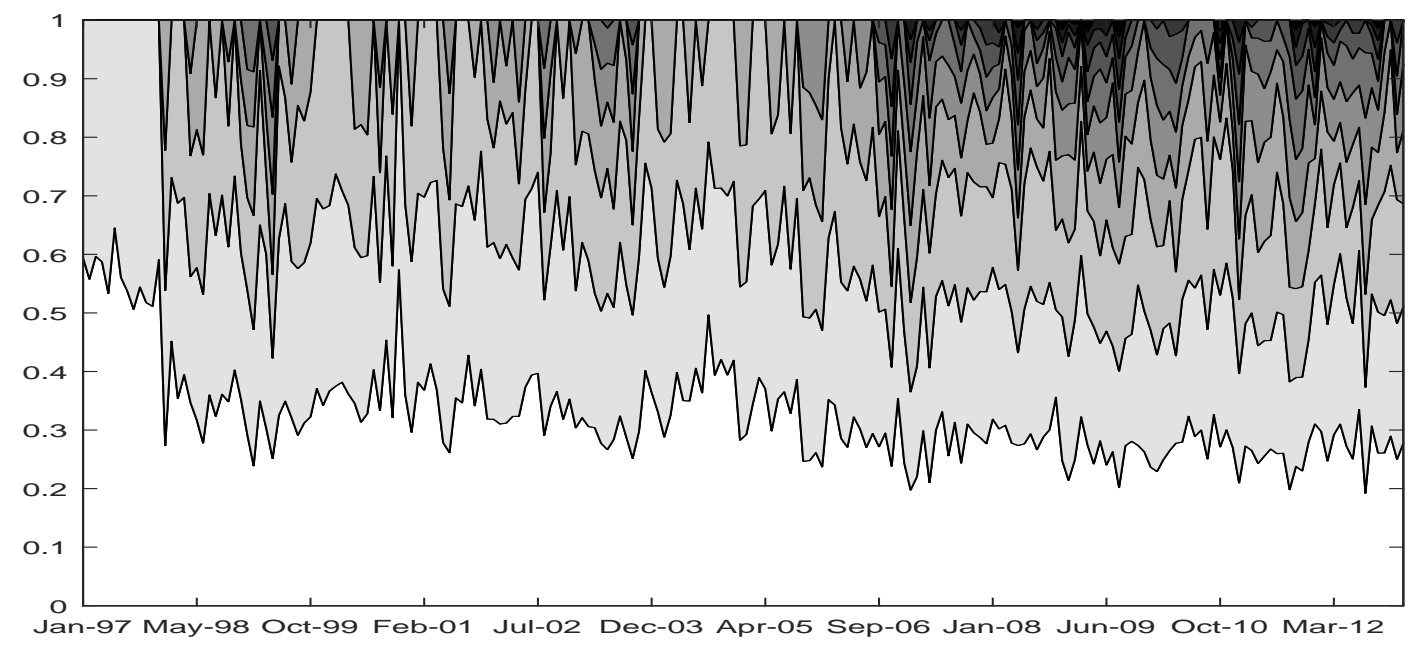

Figure 3: Size of the European financial communities (shaded areas) during 1996-2013. Each community has a different gray level.

Finally, we report the connectivity measures on the European network as presented in subsection 3.1. Figure 4 reports intra, inter communities and total network density measures from Equation 17. The empirical correlations are $\hat{\rho}_{\text {intra,total }}(0.50), \hat{\rho}_{\text {inter,total }}(0.97)$ and $\hat{\rho}_{\text {intra,inter }}(0.30)$. As shown graphically and from correlations, the total network density $(f)$ is driven by the inter-community connectivity $\left(f^{I N T E R}\right)$ with a residual role played by the intracommunity one $\left(f^{I N T E R}\right)$. This suggests a disassortativity structure of the network where the edges existing between the groups and thus, the community bridges, play a relevant role in the spread of contagion in the financial system. As further confirmation, Figure 5 shows that the relative weight of intra community (dashed line) on the total network density is lower with respect to the one of the inter community (solid line). Since the network exhibits a strong interdependent community structure, the identification of the SIFIs cannot be reduced in terms of total connectedness but should instead investigated discriminating among intra and inter community linkages. For sake of brevity, we report the other connectivity measures such as community intra and inter, in and out degrees in the Appendix E 


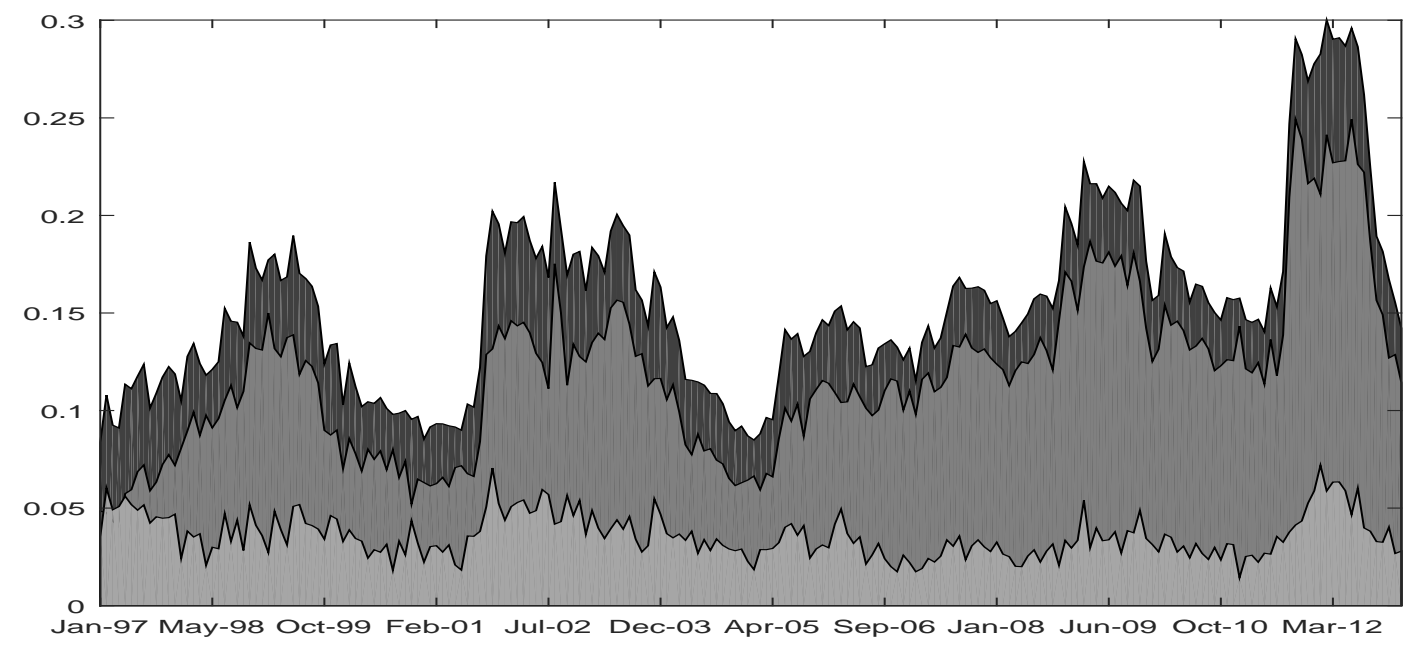

Figure 4: Density measures of the European network for intra community density (light grey), inter community density (grey) and total network density (dark grey) during 1996-2013.

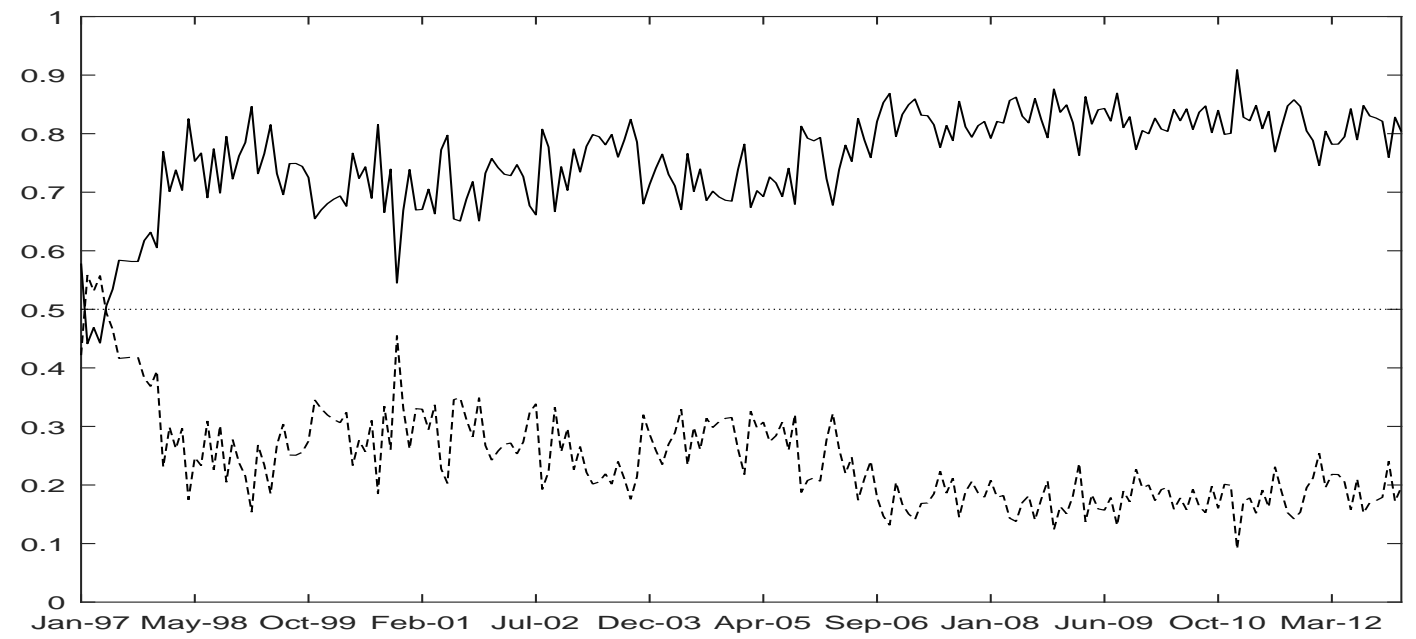

Figure 5: Relative weights of intra community (dashed line) and inter community (solid line) on the total network density during 1996-2013. 


\subsection{The Global Financial Crisis and the European Sovereign Debt Crisis}

In this subsection, we analyse the European financial network and its community structure during the Global financial crisis and the European sovereign debt crisis. Notably, according to causality, we can distinguish a community as a shock transmitter (the out inter community degrees) and as a shock receiver (the in inter community degrees). The systemically importance of a financial community (SIFC) is given by its role in the spread of contagion in the network, that is, the higher out inter community degrees net of the in inter community degrees with respect to the other communities. First, we analyse the European financial network during the global financial crisis occurred from 2007 to 2008 which has been originated from the subprime mortgage market in the United states (Shiller, 2012). We select from the analysis the adjacency matrix on November 2008 which corresponds to the $155^{\text {th }}$ window on the dynamic networks. Figure 6 includes the network with the community structures (ten communities). Panel (a) shows the directed network graph where edges are concave and clockwise directed while Panel (b) shows the adjacency matrix according to the community membership. The network is composed by 365 financial institutions: 62 Banks (red nodes), 39 Insurances (blue nodes), 84 Real Estate companies (green nodes) and 180 financial services institutions (black nodes) according to the ICB classification. The majority of the financial institutions are traded in the United Kingdom (186), Italy (50), Germany (37) and France (30).

The total density of the network is $20.44 \%$ are reported in Table 1 . Block 7 is the most systemically important financial community since it exhibits the highest net out-inter community density (7.91\%), fnet,INTRA. The network exhibits a two core structure where one core (Block 7) spread shocks almost exclusively to another core (Block 6). The inter-linkages among the two communities represent ideally a bipartite network. Block 7 is composed by Insurances $(53.85 \%$ of the market), Banks (29.03\%), Real Estate (26.19\%) and Financial Services (16.11\%) as reported in Table 3 . It is worth noting that this block includes 6 out 10 (3 banks and 3 insurances) of the largest financial institution in terms of market value Figure 6(a) and represents the largest community in terms of total market value as reported in Table 2, Block 6 is the community that largely receive shocks from Block 7 (0.0561) and for the residual part by Block 9 (0.0162) as shown in Table 4 .

Table 3 reports the European financial institutions according to the community membership at a sub-sector level where the percentages describe the weight of a community for a given sector. The community spreader (Block 7) contains the majority of the insurances in the market $(53.85 \%)$ with nonlife insurances (33.33) and life insurances (20.51\%). This is particularly interesting 


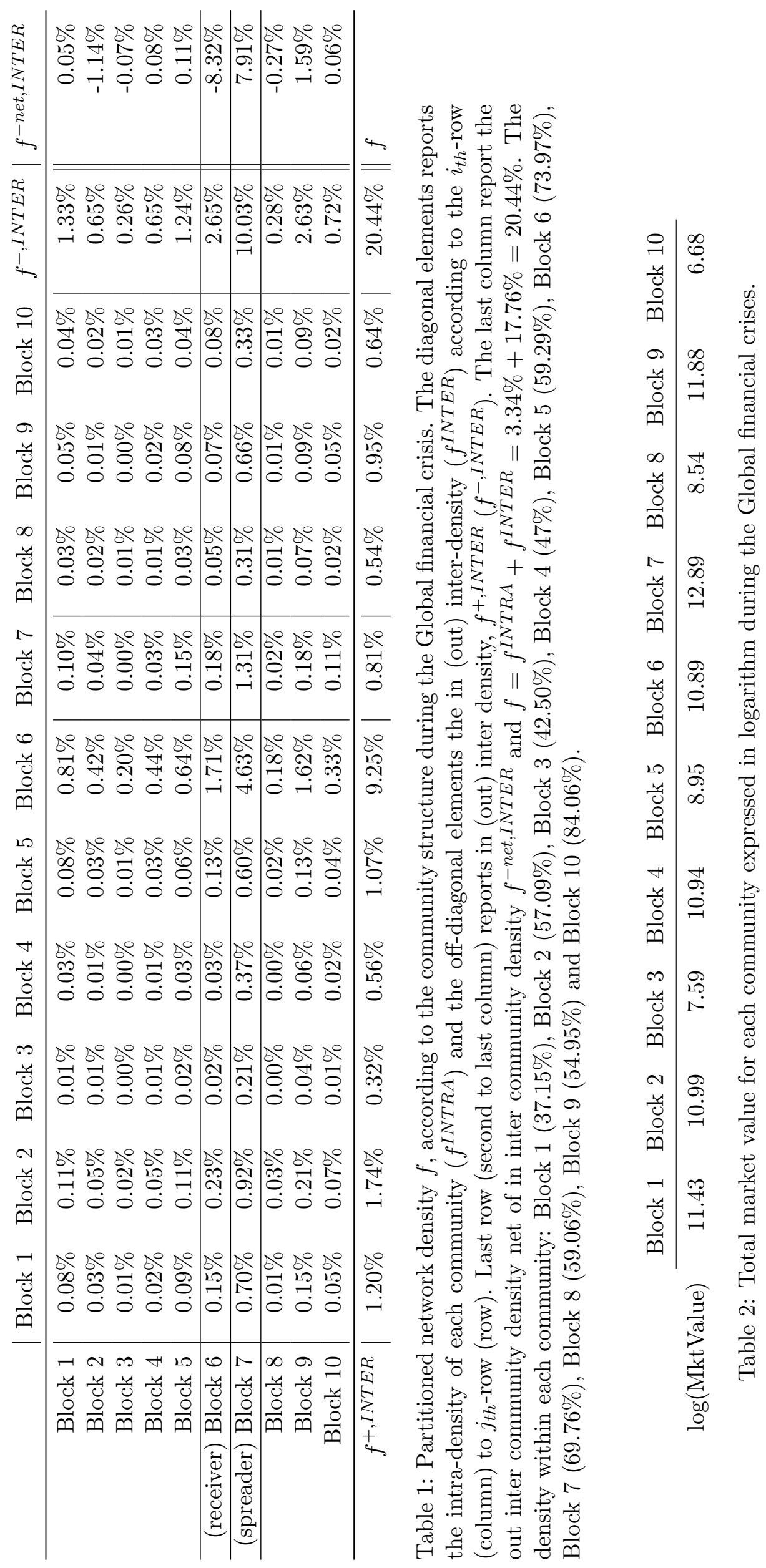




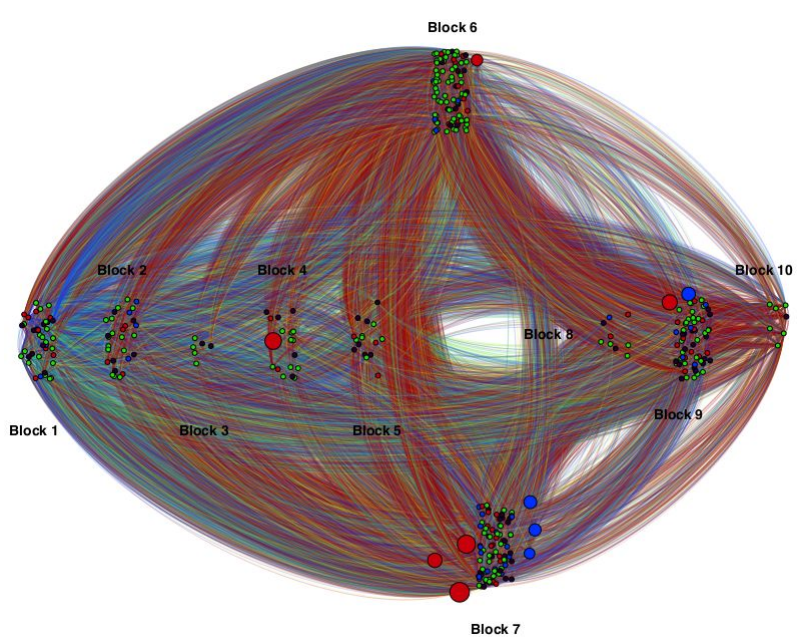

(a) Causality Network.

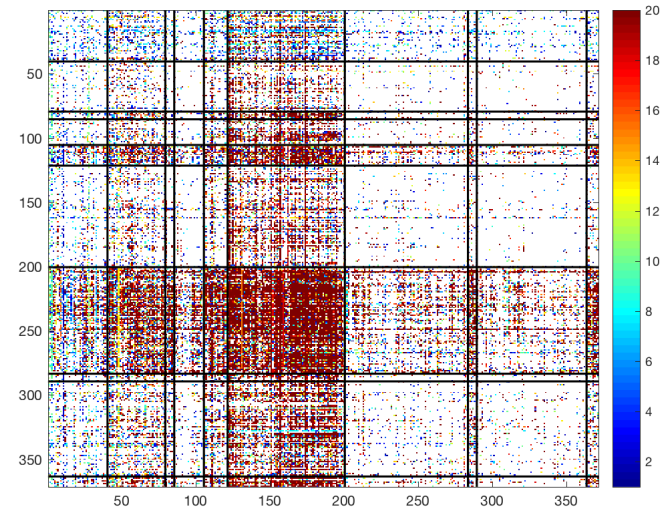

(b) Adjacency matrix.

Figure 6: The European financial network $\left(155^{\text {th }}\right.$ window) during the global financial crisis. Edges color is reported according to the edges weight (see the colormap) while nodes color is reported according to the ICB: banks (red), insurances (blue), financial services (green) and real estate (black). Panel (a): Edges are concave and clockwise directed where the size of nodes is reported according to the market value for the top 10 largest financial institution. Panel (b): Causality is meant from row $i$ to column $j$.

since it provides non-obvious evidence (Jobst, 2014$)$ on the role played by the insurance sector in propagating systemic risk. The receiver block (Block 6) contains the largest group of institutions that are in Financial services sector (35\%) with a majority of equity investment instruments $(26.11 \%) \cdot{ }^{4}$

The Global financial crisis is considered the trigger for the European sovereign debt crisis occurred at the end of 2009 since it provided a change in the asset prices and grow prospectives. Lane (2012) provides an exhaustive analysis about the pre-crisis risk factors and the relationship among the global financial crisis and the sovereign debt crisis. We select in the analysis the adjacency matrix on April 2012 which corresponds to the $199^{\text {th }}$ window od the dynamic networks. Figure 7 includes the the community structures (eight communities). Panel (a) shows the directed network graph where edges are concave and clockwise directed while Panel (b) shows the adjacency matrix according to the community membership. The network is composed by 359 financial institutions: 58 Banks (red nodes), 33 Insurances (blue nodes), 82 Real Estate companies (green nodes) and 186 financial services institutions (black nodes) according to the ICB. The majority of the financial institutions are traded in the United Kingdom (173), Germany

\footnotetext{
${ }^{4}$ According to the Industrial Classification Benchmark, the Equity investment instruments sub-sector is composed by corporate closed-ended investment entities identified under distinguishing legislation, such as investment trusts and venture capital trusts.
} 


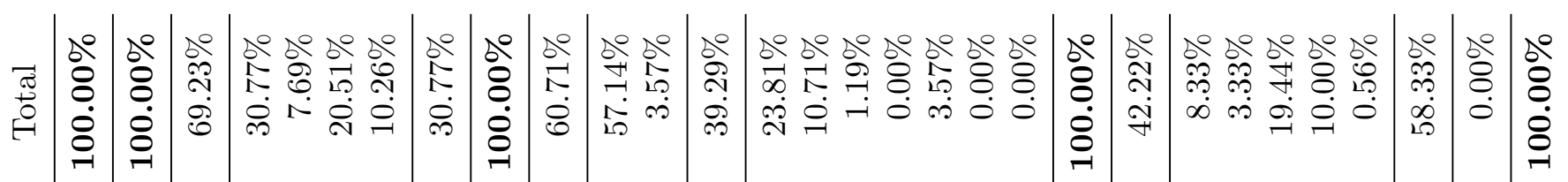

군

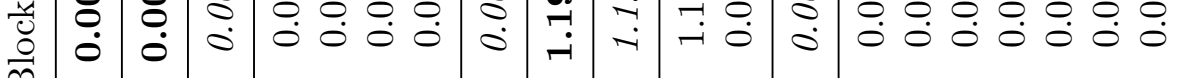

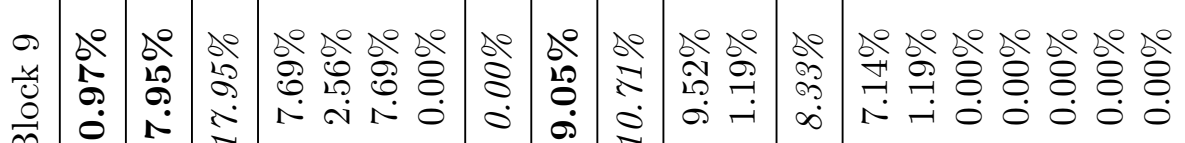

ต

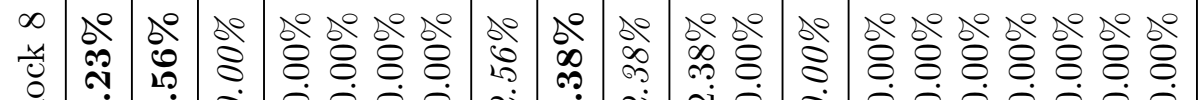

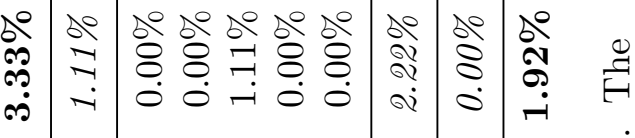

ำ

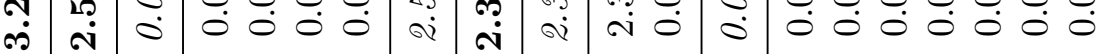

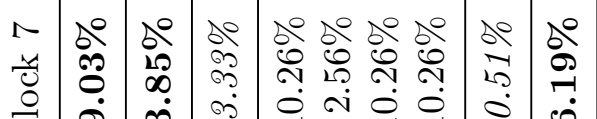

虫

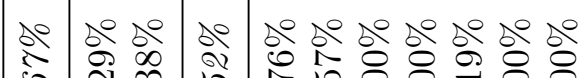

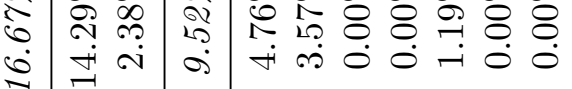

0

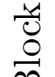

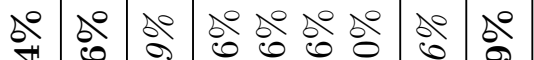

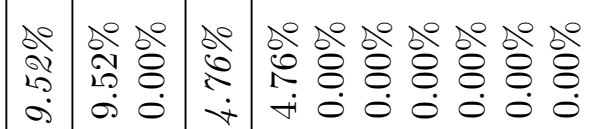

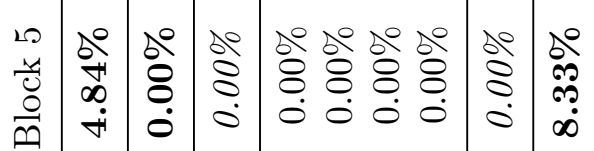

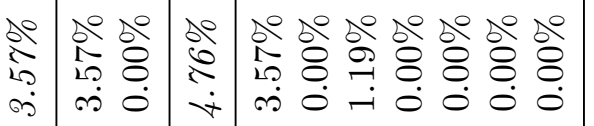

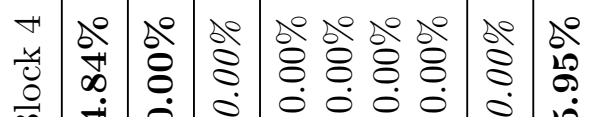

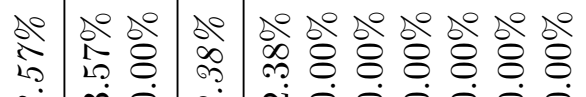

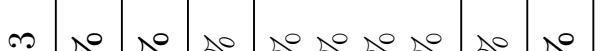

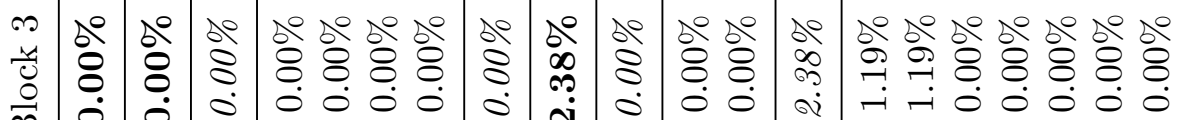

m

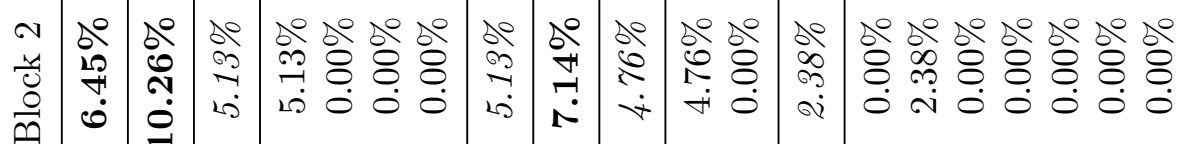

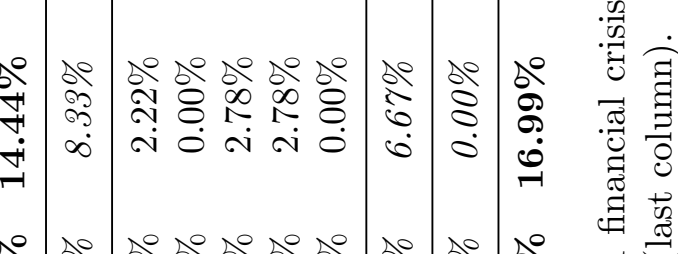

ฟิ

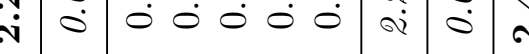

- to jo jo do do y

$\bar{m}$

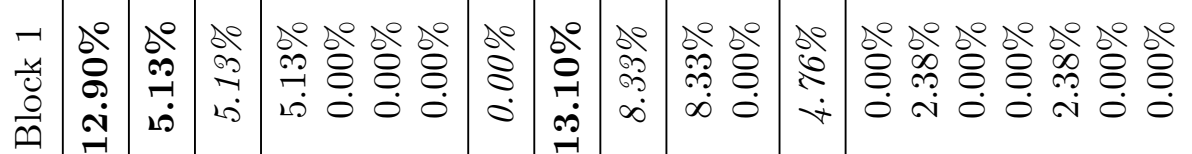

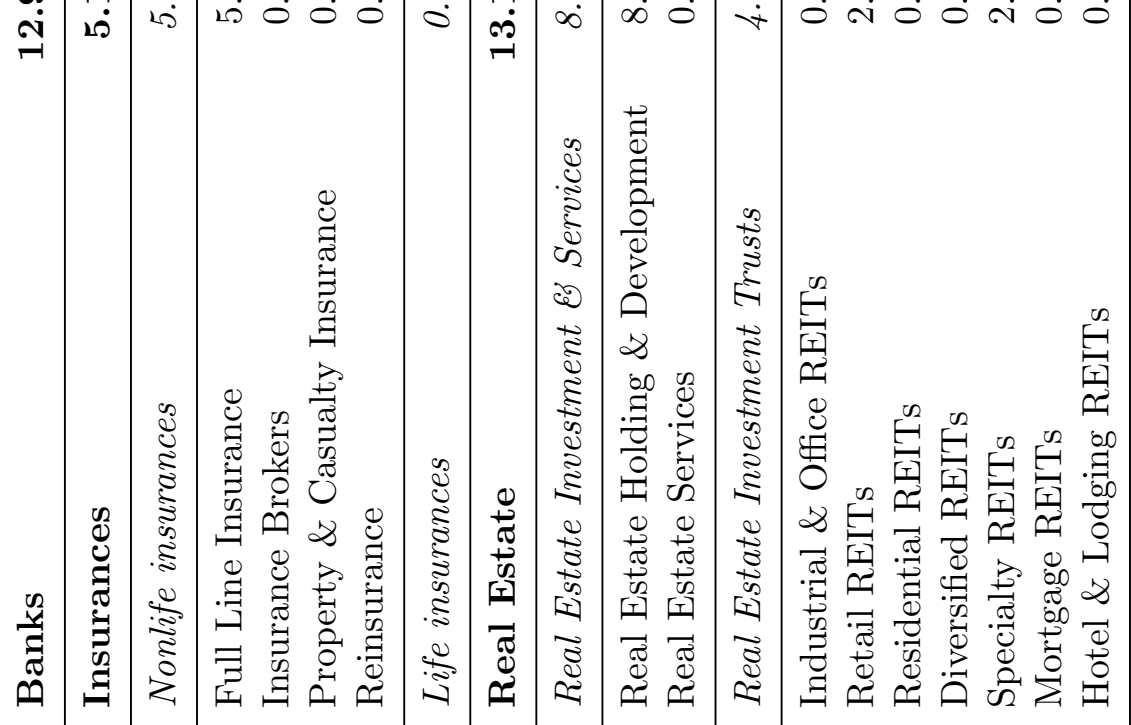

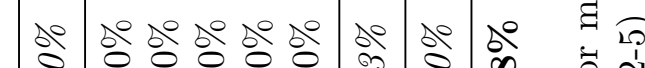

\& 8 \& 8 \& 8 :

$\begin{array}{lllllllllll}0 & 0 & 0 & 0 & 0 & 0 & \infty & 0 & 0 & 0\end{array}$

要

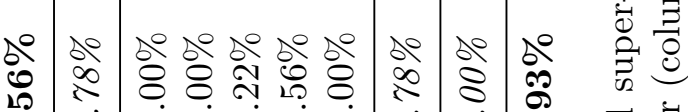

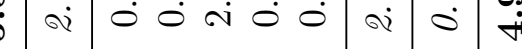

㞼

พิ|

द्व

- so je jo jo so so

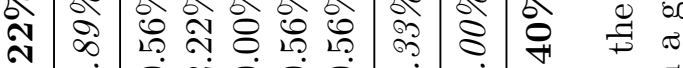

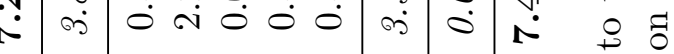


(58), Italy (44) and France (34) the majority of the markets.

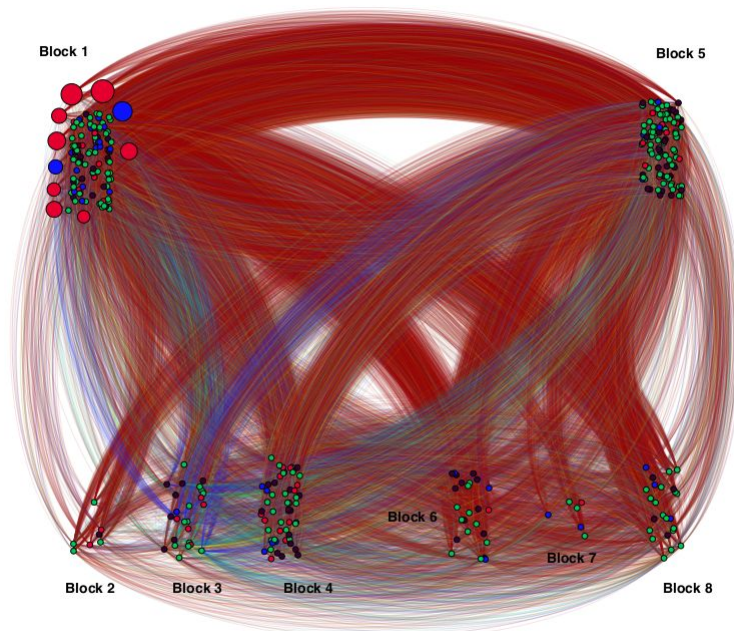

(a) Causality Network.

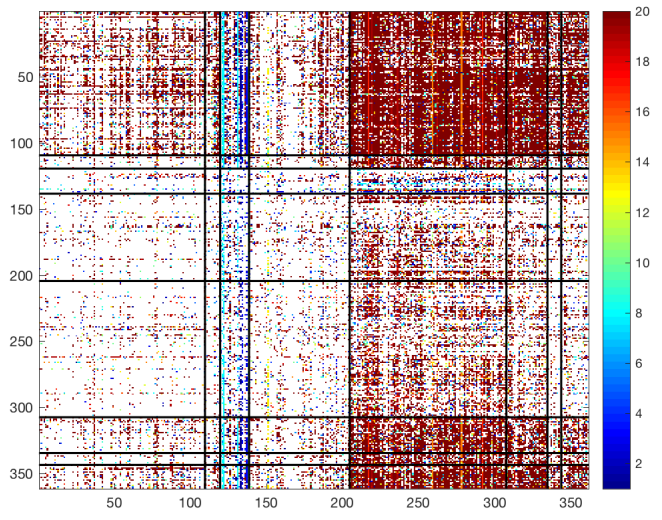

(b) Adjacency matrix.

Figure 7: The European financial network $\left(199^{\text {th }}\right.$ window) during the European sovereign debt crisis. Edges color is reported according to the edges weight (see the colormap) while nodes color is reported according to the ICB: banks (red), insurances (blue), financial services (green) and real estate (black). Panel (a): Edges are concave and clockwise directed where the size of nodes is reported according to the market value for the top 10 largest financial institution. Panel (b): Causality is meant from row $i$ to column $j$.

As shown in Table 4, the total density of the network is $29.11 \%$. Block 1 is the most systemically important financial community since it exhibits the highest net out-inter community density (10.71\%), $f^{n e t, I N T R A}$. The network exhibits a two core structure where one core (Block 1) spread shocks mainly to other core (Block 5$)$. Block 1 is composed by Insurances $(51.52 \%$ of the market), Banks (39.66\%), Financial Services (30.65\%) and Real Estate (19.51\%) as reported in Table ?? It is worth noting that this block includes all the top ten financial institutions in terms of market value ( 8 banks and 2 insurances). This Block represents also in this case the largest community in terms of market value (Table 4). Block 5 is the community that largely receive shocks from Block 1 (7.15\%) and for a residual part mainly by Block 4, Block 6 and Block 8 Table 4. Table ?? reports the European financial institutions according to the community membership at a sub-sector level where the percentages describe the weight of a community for a given sector. Also in this case, the community spreader (Block 1) contains the majority of the insurances in the market $(51.52 \%)$ with nonlife insurances (27.27) and life insurances (24.24\%). This highlights that the insurance industry played a prominent role in the spread of systemic risks even in the European sovereign debt crisis in the same manner as in the Global financial crisis. The receiver Block 5 contains the largest group of institutions that are 
in Financial services sector (34.41\%) and Real Estate sector (34.15\%) with almost equal weights at their sub-sector level.

\section{$5 \quad$ Financial Bridges and Policy Implications}

\subsection{Early Warning Indicators}

As seen in Section 4.2, the density of the network is driven mainly by the inter-community connections which highlights the role of financial community bridges in the spread of contagion. Following Billio et al. (2012) and Acharya et al. (2017), we perform an out-of-sample analysis of the community measures with respect to the usual network measures. We compare their predictive ability in terms of future losses. In this regard, we define the variable MaxLoss, the maximum loss incurred by a single institution within the next two years and use total out and inter- (intra-) community out degrees as predictors in the linear model $]^{5}$

$$
\operatorname{MaxLos}_{i}=c+\beta X_{i}+\varepsilon_{i}, \quad \varepsilon_{i} \stackrel{i i d}{\sim}\left(0, \sigma^{2}\right)
$$

$i=1, \ldots, n$ where $X_{i}=\left\{d_{i}^{-}, d_{i}^{-, I N T E R}, d_{i}^{-, I N T R A}\right\}$ is expressed in relative value to make the coefficients comparable. We perform the cross-sectional regressions sequentially from 2000 to 2012 and report in Figure 8 the regression adjusted R-squared, and the magnitude and p-value of each coefficient.

The out-inter community degrees (dotted line) provide the best fitting over the total out degrees (solid line) more than the $93 \%$ of the total cases while the intra-community in degrees (dashed line) provide the lowest performance in every period. The p-value analysis shows a significance at $5 \%$ confidence level of $79 \%$ for the total out degrees (solid line), $85 \%$ for out-inter community degrees (dotted line) and $13 \%$ for the in-intra community degrees (dashed line). Finally, the coefficient is negative in more than $99 \%$ of the total significant cases which further indicates that the nodes with the highest out degrees incur to a lower loss with respect to the other nodes which indicates and consequently, they represent the most resilient institutions. The order of magnitude is always greater for the inter-community out degrees (dotted line) with respect to the total out degrees (solid line). These findings conform the primary role of the community inter-linkages in spreading shocks and hence, an early warning indicator for financial losses in the system. they represent the most critical institutions in the spread of losses in the system.

\footnotetext{
${ }^{5}$ We also perform the analysis for the in connections. Results are available upon request to the authors.
} 


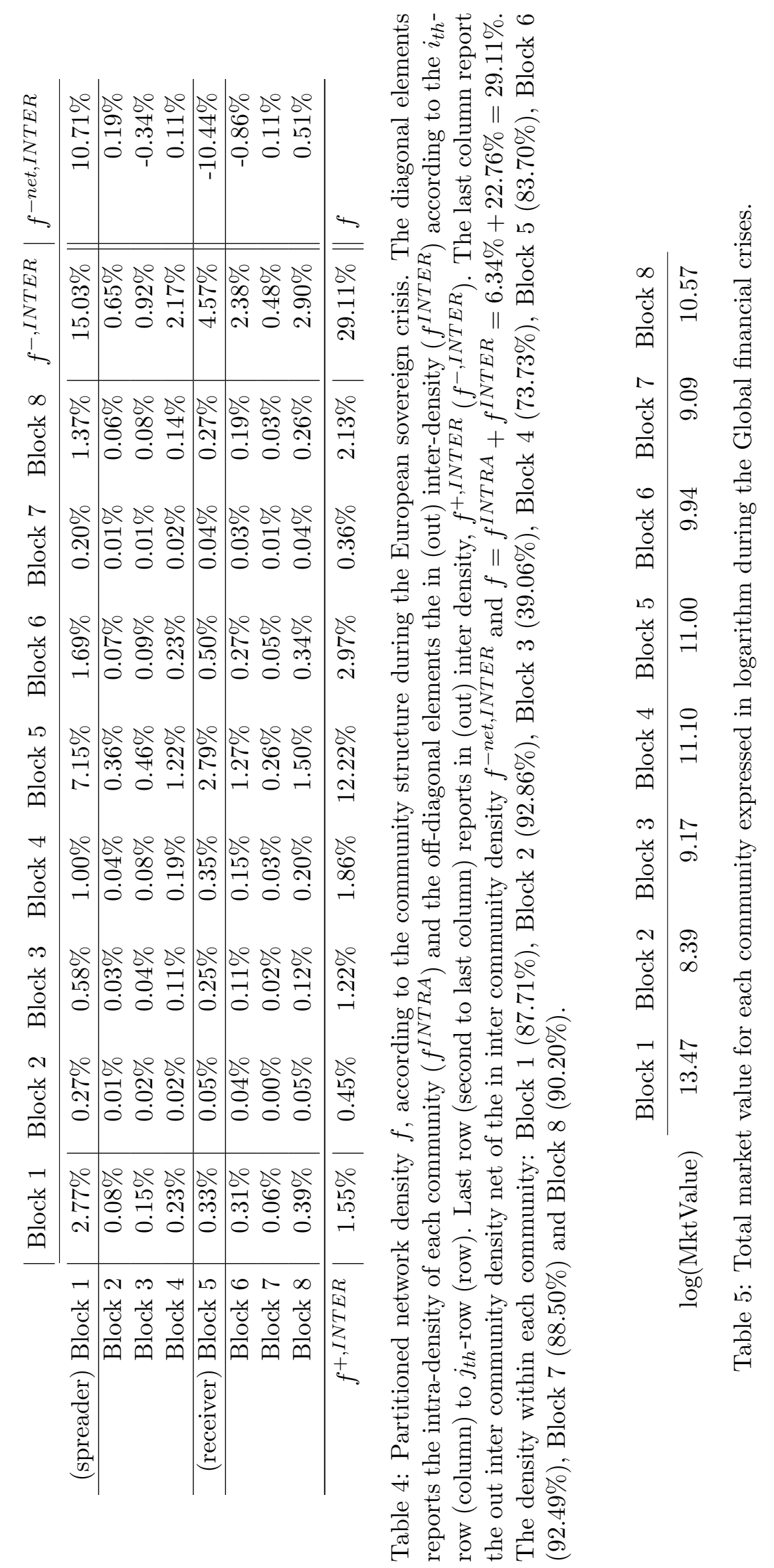




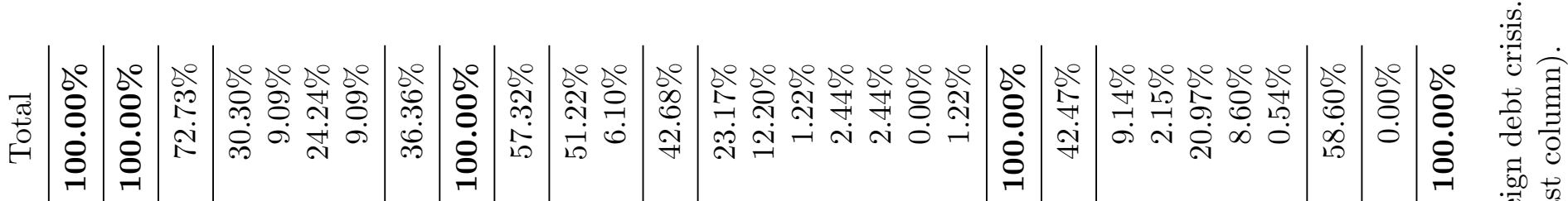

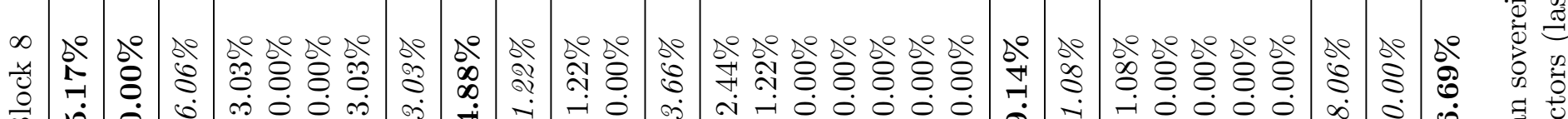

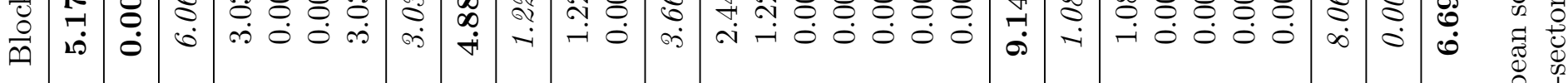

ᄂ

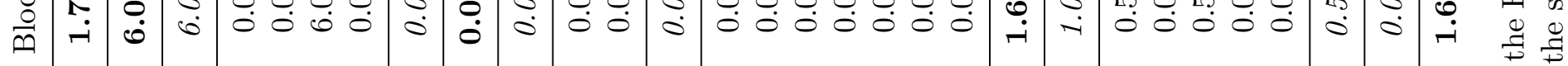

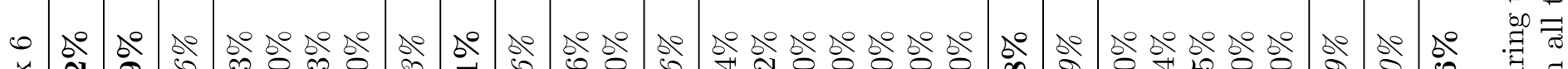

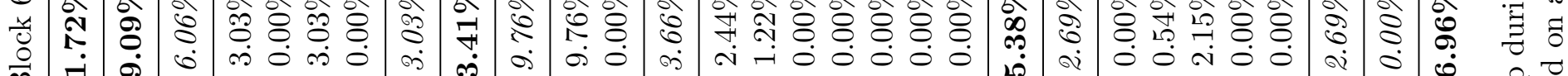

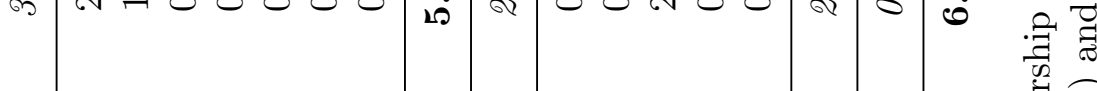

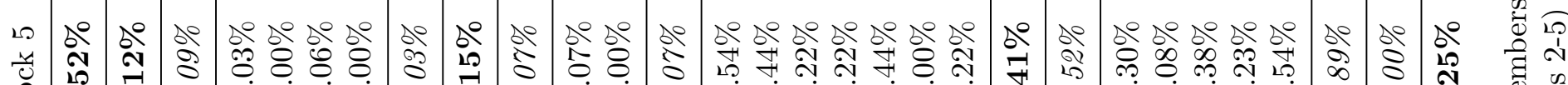

昰

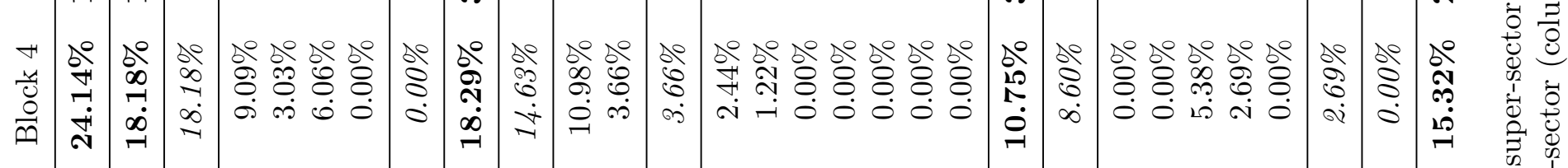

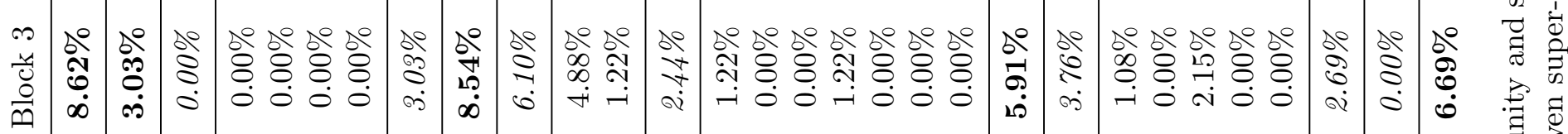

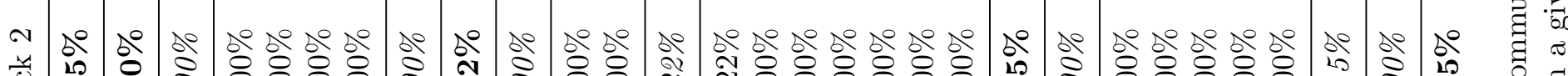

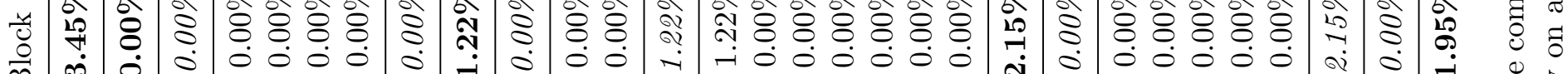

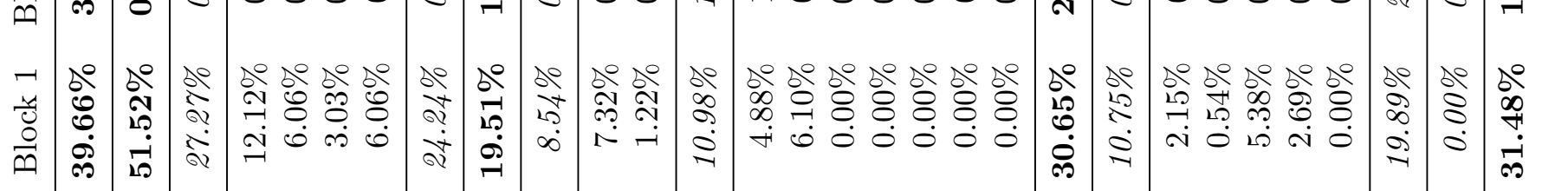

秀.

$\circ$ 코

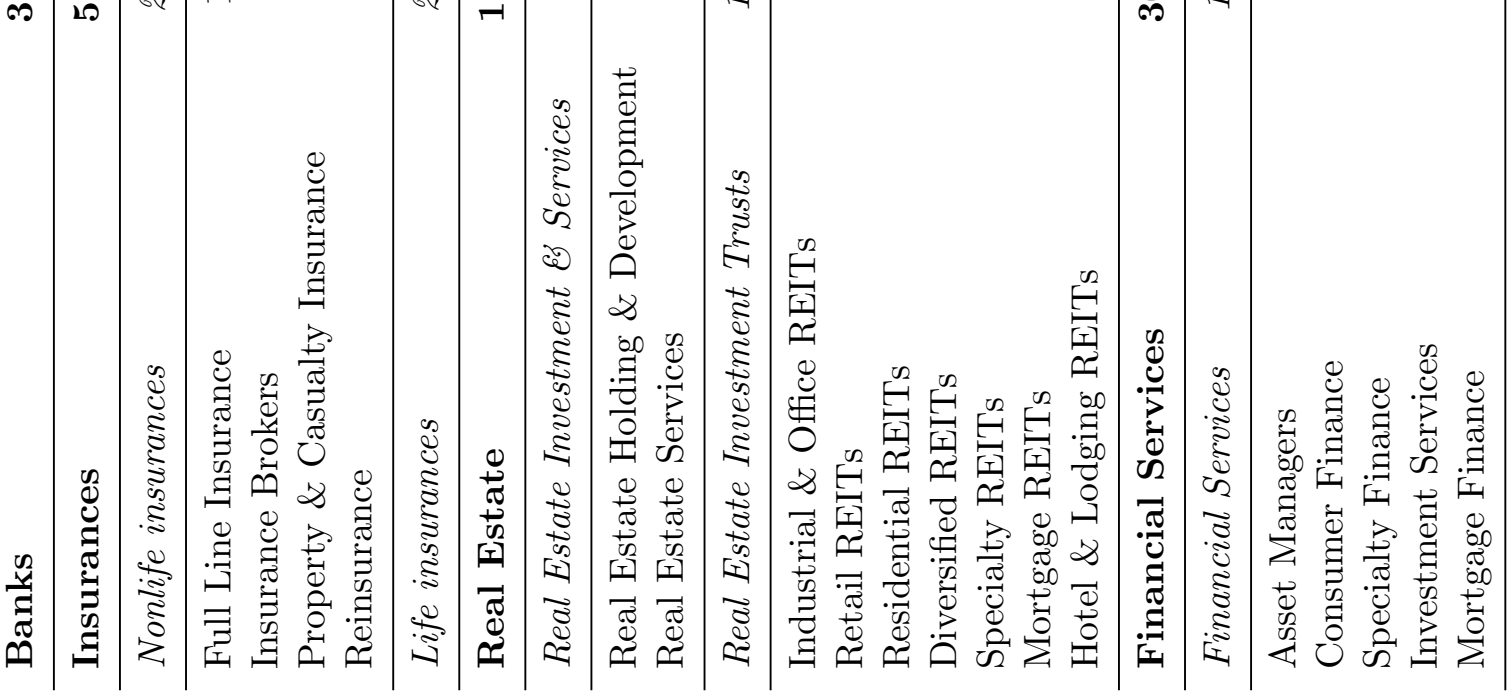

$\exists$ ठ

过

䓪

壱

要这

.

敢

สี

응

牙芯 
The order of magnitude is always greater for the inter-community out degrees (dotted line) with respect to the total out degrees (solid line). The findings confirm the primary role of the community inter-linkages as early warning indicator for financial losses. As further comparison, we include in Appendix F the regression with the eigenvector centrality measure which confirms the previous results.

\subsection{Network Immunization}

Policy authorities act through prevention and mitigation measures in order to maintain financial stability. When a systemic risk arises in the network, policy interventions should immunize the node-spreaders in order to avoid the financial contagion. As stated in Salathé and Jones (2010), the presence of a community structure significantly affects the dynamic of the disease and in such cases, immunization interventions should focus on community bridges instead of the nodes which are highly connected to the whole network.

In this regard, a node immunization is obtained recursively by switching off the node with: i) the highest total out degrees (global immunization); ii) the highest eigenvector centrality (centrality immunization) and iii) the highest community inter out degrees (community immunization). We make use of the average shortest path length (ASPL) as the evaluation criteria to compare the goodness of the two immunization strategies. ASPL measures the average number of steps along the shortest paths for all possible pairs of network nodes,

$$
A S P L_{t}=\left(n_{t}\left(n_{t}-1\right)\right)^{-1} \sum_{i, j}^{n_{t}} d(i, j),
$$

where $n_{t}$ is the number of nodes in the network and $d(i, j)$ is the shortest path from node $i$ to node $j$. In a financial network, ASPL indicates the number of financial institutions that a single institution have to affect on average in order to transmit financial contagion to a not connected institution.

After the immunization of a node, the mitigation of systemic risk succeeds if there is an increase on the ASPL of the network. That is, the number of steps on average increases and thus, network becomes more robust to shock propagation. Conversely, if ASPL decreases, the intervention is not only useless, but harmful since the network is more exposed to financial instability.

As an example for policy intervention, we perform the analysis for the three type of immunizations on the financial network during the European sovereign debt crisis where the given crisis has been originated (199 ${ }^{\text {th }}$ window). Figure 9 shows that the resulting ASPL after removing 


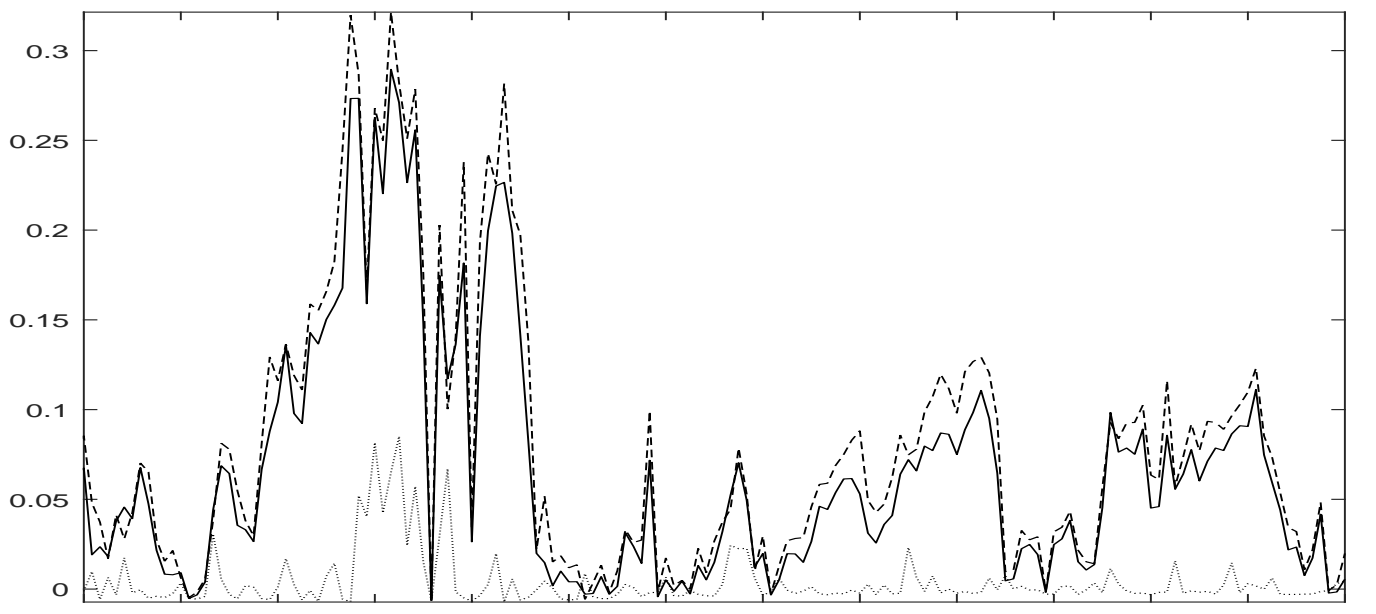

Feb-00Jan-01Dec-01Nov-02Oct-03Sep-04Aug-05 Jul-06 Jun-07May-08Apr-09Mar-1 0Feb-11 Jan-12

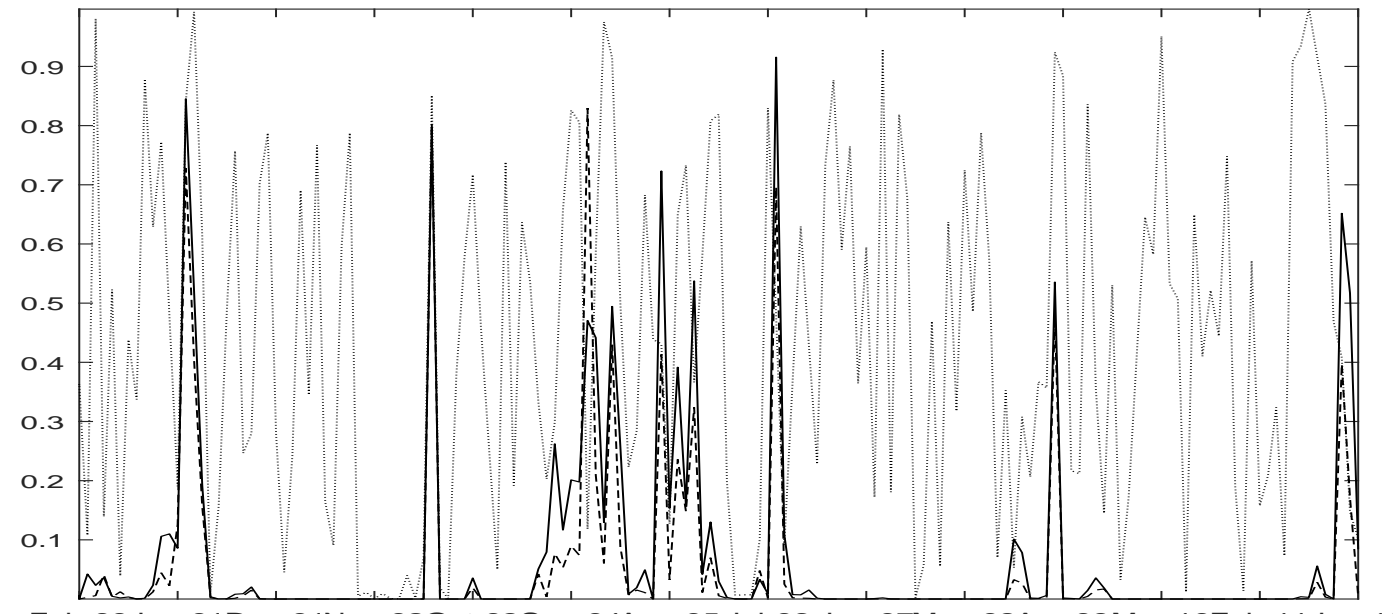

Feb-00Jan-01 Dec-01Nov-02Oct-03Sep-04Aug-05 Jul-06 Jun-07May-08Apr-09Mar-1 0Feb-11 Jan-12

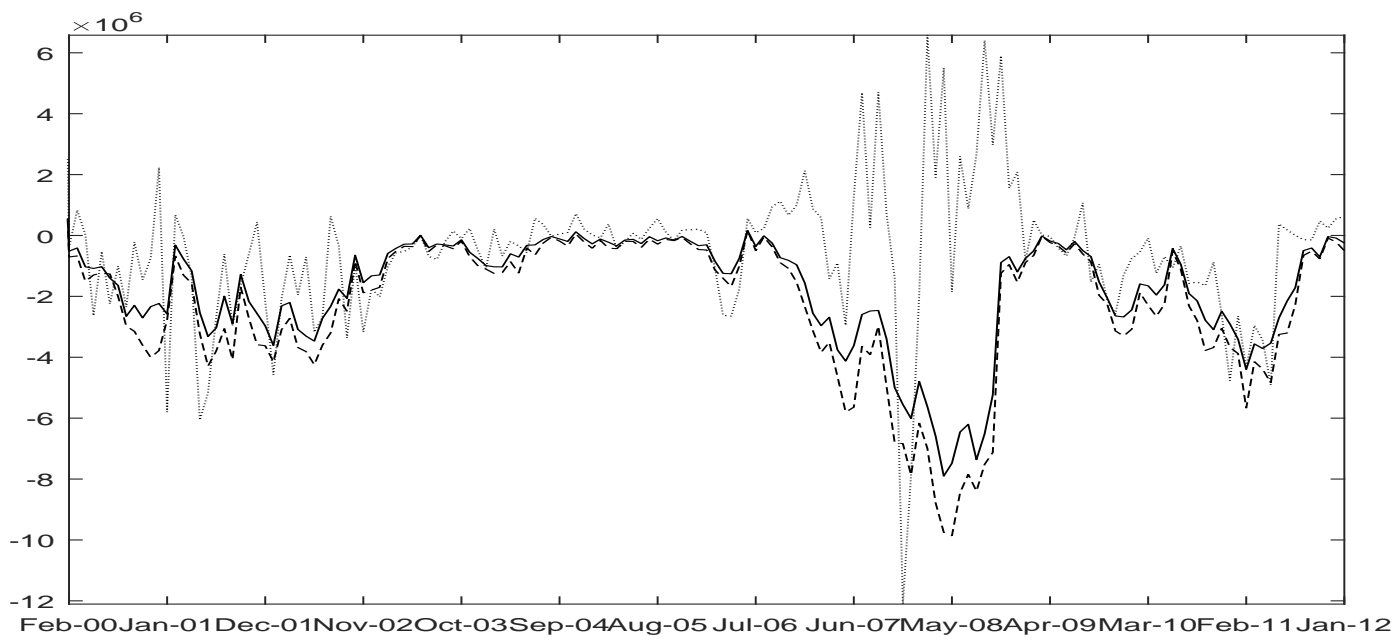

Figure 8: Adjusted R-squared (top), coefficient p-value (mid) and coefficient magnitude (bottom) for the cross-sectional regressions where the dependent variable is the maximum loss incurred by a single institution within the next two years and the independent variable is alternatively the total out degrees (solid line), the out-inter community degrees (dashed line) and the out-intra community degrees (dotted line). 
up to 15 nodes in the network with the highest community inter out degrees (dashed red line) performs overall better than the highest total out degrees (solid black line) and the highest eigenvector centrality (dotted black line). The marginal contribution of each node is higher at the beginning for the out inter community degrees with respect to the total out degrees which confirms that immunization through community bridges is more effective than usual connectedness measures. As with Salathé and Jones (2010) in epidemiology immunization, also in the financial system the results in terms of public intervention are more efficient through treatments adopted for community bridges. In fact, community inter out degrees converges towards an inverse ASPL value of 4.25 after the immunization of 15 nodes while the total out degrees and the eigenvector centrality reach a value of 4.19 and 4.20 , respectively ${ }^{6}$

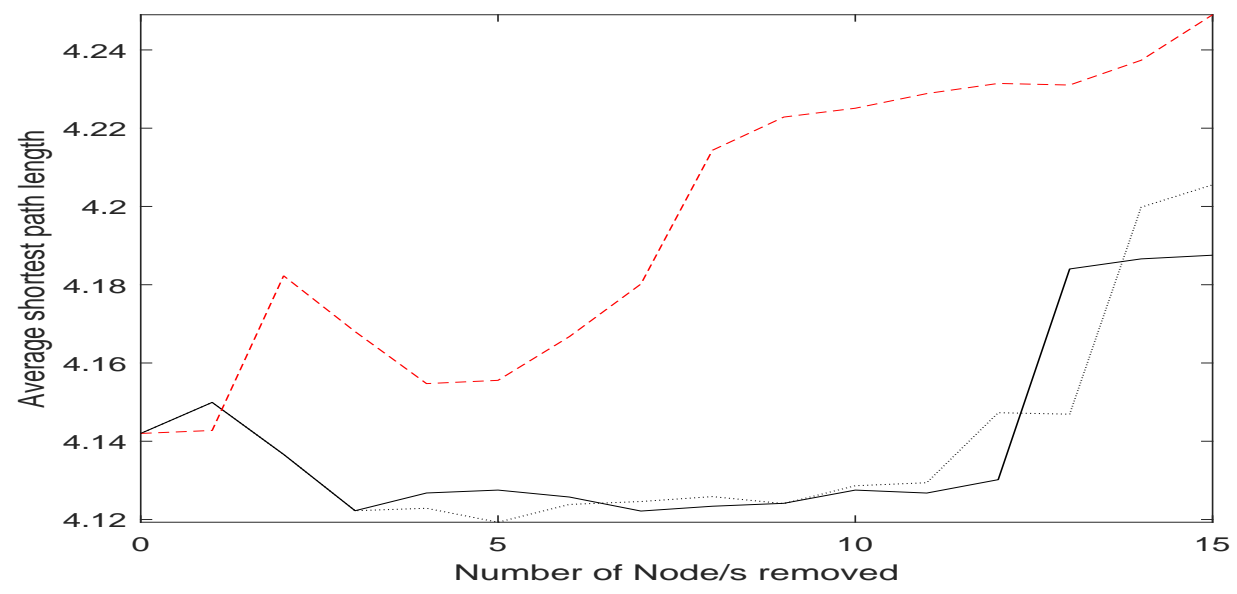

Figure 9: Average shortest path length (ASPL) after removing sequentially the node(s) with the highest total out degrees (solid black line), the highest eigenvector centrality (dotted black line) and the highest inter-community out degrees (dashed red line).

\section{Conclusion}

The recent financial crises show the need for a deeper investigation of the structure of the financial system. The notion of networks have been successfully used in the analysis of financial stability. We show that financial networks exhibit a local community structure and a number of institutions acting as bridges between financial communities. This occurs also in many real networks which often display inhomogeneity not only globally but also locally, with the concentration of edges within groups of nodes and low concentrations between groups or with groups of nodes sharing similar connectivity patterns in the network (Newman, 2006). We identify the

\footnotetext{
${ }^{6}$ As robustness check, we compute ASPL on all the period (210 networks) after removing 25 nodes. ASPL is higher for the community inter out degrees more than the $67 \%$ of the cases.
} 
communities through stochastic block models and propose new measures of community connectedness based on the interdependence within and between the communities. Our findings show that during the last financial crises, the European financial network exhibits a core-periphery structure with the presence of a main block acting as shock spreader to the rest of the network. We define this kind of blocks as systemically important financial communities. Furthermore, we find an increase of the number of communities during periods of financial distress, a signal of network disintegration in different sub-blocks with the rise of heterogeneous connectivity patterns. Consequently, the node immunization based on the identification of highly connected nodes may be less effective in a network showing a strong community structure. In this regard, we perform an immunization exercise during the European sovereign debt crisis. Results show that removing the institutions with highest inter-community degrees leads to a reduction in contagion effect, i.e. it increases significantly the average shortest path length between nodes. Thus, monitoring and adopting treatments for financial bridges would represents a more efficient mitigation policy than the one based on total degree connectedness measures. Moreover, we find that inter community connectedness measures represent a good early warning indicator for future financial losses which can increase the regulators' and policy makers ability to respond promptly to abrupt changes of an evolving financial system. A further confirmation that financial bridges play a primary role in the spread of financial contagion.

\section{References}

Acemoglu, D., Ozdaglar, A., and Tahbaz-Salehi, A. (2015). Systemic risk and stability in financial networks. American Economic Review, 105(2):564-608.

Acharya, V. V., Pedersen, L. H., Philippon, T., and Richardson, M. (2017). Measuring systemic risk. Review of Financial Studies, 30(1):2-47.

Adrian, T. and Brunnermeier, M. K. (2016). CoVaR. American Economic Review, 106(7):170541.

Ahelegbey, D. F., Billio, M., and Casarin, R. (2016a). Bayesian graphical models for structural vector autoregressive processes. Journal of Applied Econometrics, 31(2):357-386.

Ahelegbey, D. F., Billio, M., and Casarin, R. (2016b). Bayesian graphical models for structural vector autoregressive processes. Journal of Applied Econometrics, 31(2):357-386. 
Ahelegbey, D. F., Billio, M., and Casarin, R. (2016c). Sparse graphical vector autoregression: A Bayesian approach. Annals of Economics and Statistics, 123/124:333-361.

Aicher, C., Jacobs, A. Z., and Clauset, A. (2014). Learning latent block structure in weighted networks. Journal of Complex Networks, 3(2):221-248.

Allen, F. and Carletti, E. (2013). What is systemic risk? Journal of Money, Credit and Banking, 45(s1):121-127.

Allen, L., Bali, T. G., and Tang, Y. (2012). Does systemic risk in the financial sector predict future economic downturns? Review of Financial Studies, 25(10):3000-3036.

Bargigli, L. and Gallegati, M. (2013). Finding communities in credit networks. Economics, $7(17): 1$.

Barigozzi, M. and Brownlees, C. T. (2016). Nets: Network estimation for time series. Available at SSRN 2249909.

Bates, J. M. and Granger, C. W. (1969). The combination of forecasts. Journal of the Operational Research Society, 20(4):451-468.

Billio, M., Costola, M., Panzica, R., and Pelizzon, L. (2017). Systemic risk and financial interconnectedness: network measures and the impact of the indirect effect. In Billio, M., Pelizzon, L., and Savona, R., editors, Systemic Risk Tomography: Signals, Measurement and Transmission Channels, chapter 2, pages 43-72. iSTE Press - Elsevier.

Billio, M., Getmansky, M., Lo, A. W., and Pelizzon, L. (2012). Econometric measures of connectedness and systemic risk in the finance and insurance sectors. Journal of Financial Economics, 104(3):535-559.

Blei, D. M., Kucukelbir, A., and McAuliffe, J. D. (2017). Variational inference: A review for statisticians. Journal of the American Statistical Association.

Board, F. S. (2009). Guidance to assess the systemic importance of financial institutions, markets and instruments: initial considerations. Report to G20 finance ministers and governors.

Brownlees, C. and Engle, R. F. (2017). SRISK: A conditional capital shortfall measure of systemic risk. Review of Financial Studies, 30(1):48-79. 
De Souza, S. R. S., Silva, T. C., Tabak, B. M., and Guerra, S. M. (2016). Evaluating systemic risk using bank default probabilities in financial networks. Journal of Economic Dynamics and Control, 66:54-75.

Diebold, F. X. and Yilmaz, K. (2014). On the network topology of variance decompositions: Measuring the connectedness of financial firms. Journal of Econometrics, 182(1):119-134.

Diebold, F. X. and Yilmaz, K. (2015). Financial and Macroeconomic Connectedness: A Network Approach to Measurement and Monitoring. Oxford University Press.

Elliott, M., Golub, B., and Jackson, M. O. (2014). Financial networks and contagion. The American economic review, 104(10):3115-3153.

Fortunato, S. (2010). Community detection in graphs. Physics Reports, 486(3):75-174.

Franklin Allen, A. B. (2009). Networks in finance. In Kleindorfer, P. and Wind, Y., editors, The Network Challenge: Strategy, Profit, and Risk in an Interlinked World, chapter 21, pages 367-382. Pearson Prentice Hall.

Freixas, X., Parigi, B. M., and Rochet, J.-C. (2000). Systemic risk, interbank relations, and liquidity provision by the central bank. Journal of Money, Credit and Banking, 32(3):611638.

Giglio, S., Kelly, B., and Pruitt, S. (2016). Systemic risk and the macroeconomy: An empirical evaluation. Journal of Financial Economics, 119(3):457-471.

Hałaj, G. and Kok, C. (2013). Assessing interbank contagion using simulated networks. Computational Management Science, 10(2-3):157-186.

Haldane, A. G. (2013). Rethinking the financial network. In Fragile stabilität-stabile fragilität, pages 243-278. Springer.

Haldane, A. G. and May, R. M. (2011). Systemic risk in banking ecosystems. Nature, 469(7330):351-355.

Hautsch, N., Schaumburg, J., and Schienle, M. (2015). Financial network systemic risk contributions. Review of Finance, 19(2):685-738.

Holland, P. W., Laskey, K. B., and Leinhardt, S. (1983). Stochastic blockmodels: First steps. Social Networks, 5(2):109-137. 
Hollo, D., Kremer, M., and Lo Duca, M. (2012). CISS - a composite indicator of systemic stress in the financial system. ECB Working Paper Series, 1426.

Huang, A., Wand, M. P., et al. (2013). Simple marginally noninformative prior distributions for covariance matrices. Bayesian Analysis, 8(2):439-452.

Hurd, T. (2016). Contagion! Systemic Risk in Financial Networks. SpringerBriefs in Quantitative Finance. Springer International Publishing.

Jobst, A. A. (2014). Systemic risk in the insurance sector: A review of current assessment approaches. The Geneva Papers on Risk and Insurance-Issues and Practice, 39(3):440-470.

Karrer, B. and Newman, M. E. (2011). Stochastic blockmodels and community structure in networks. Physical Review E, 83(1):016107.

Lane, P. R. (2012). The european sovereign debt crisis. The Journal of Economic Perspectives, 26(3):49-67.

Leicht, E. A. and Newman, M. E. (2008). Community structure in directed networks. Physical Review Letters, 100(11):118703.

Loepfe, L., Cabrales, A., and Sánchez, A. (2013). Towards a proper assignment of systemic risk: the combined roles of network topology and shock characteristics. PLOS One, 8(10):e77526.

McGrory, C. and Titterington, D. (2007). Variational approximations in Bayesian model selection for finite mixture distributions. Computational Statistics \& Data Analysis, 51(11):5352 $-5367$.

Newman, M. (2010). Networks: an introduction. Oxford University Press.

Newman, M. E. (2006). Modularity and community structure in networks. Proceedings of the National Academy of Sciences, 103(23):8577-8582.

Penny, W., Kiebel, S., and Friston, K. (2003). Variational Bayesian inference for fmri time series. NeuroImage, 19(3):727-741.

Pham, T. H., Ormerod, J. T., and Wand, M. P. (2013). Mean field variational Bayesian inference for nonparametric regression with measurement error. Computational Statistics $\mathcal{E}$ Data Analysis, 68:375 - 387 . 
Puliga, M., Flori, A., Pappalardo, G., Chessa, A., and Pammolli, F. (2016). The accounting network: How financial institutions react to systemic crisis. PLOS One, 11(10):e0162855.

Salathé, M. and Jones, J. H. (2010). Dynamics and control of diseases in networks with community structure. PLOS Computational Biology, 6(4):e1000736.

Scott, H. (2016). Connectedness and Contagion: Protecting the Financial System from Panics. MIT Press.

Shiller, R. J. (2012). The subprime solution: how today's global financial crisis happened, and what to do about it. Princeton University Press.

Shumway, T. (1997). The delisting bias in crsp data. The Journal of Finance, 52(1):327-340.

Teschendorff, A. E., Wang, Y., Barbosa-Morais, N. L., Brenton, J. D., and Caldas, C. (2005). A variational Bayesian mixture modelling framework for cluster analysis of geneexpression data. Bioinformatics, 21:3025-3033.

Thomson, J. B. (2010). On systemically important financial institutions and progressive systemic mitigation. DePaul Business \& Commercial Law Journal, 8:135-419.

Titterington, D. (2004). Bayesian methods for neural networks and related models. Statistical Science, pages 128-139.

Wand, M. P., Ormerod, J. T., Padoan, S. A., Fuhrwirth, R., et al. (2011). Mean field variational Bayes for elaborate distributions. Bayesian Analysis, 6(4):847-900. 


\section{A Proofs of the Results in Section 2.2}

i. Proof of the results in Eq. 9

For the observations in the sample period from $t_{m-1}+1$ to $t_{m}$ the likelihood is

$$
\begin{aligned}
\mathcal{L}\left(A_{m: m+1} \mid \mathbf{z}_{m}, \boldsymbol{\theta}_{k}\right) & =\prod_{t=t_{m-1}+1}^{t_{m}} \prod_{(i, j) \in E_{m}} \theta_{z_{i m} z_{j m}}^{A_{i j, t}}\left(1-\theta_{m, z_{i m} z_{j m}}\right)^{1-A_{i j, t}}, \\
& =\prod_{i=1}^{n} \prod_{j=1}^{n}\left(\theta_{m, z_{i m} z_{j m}}\right)^{t=t_{m-1}+1} A_{i j, t}^{t_{m}}\left(1-\theta_{m, z_{i m} z_{j m}}\right)^{t=t_{m-1}+1} \sum^{t_{m}}\left(1-A_{i j, t}\right)
\end{aligned}
$$

which can be written in exponential form as

$$
\begin{aligned}
\mathcal{L}\left(A_{m: m+1} \mid \mathbf{z}_{m}, \boldsymbol{\theta}_{k}\right) & =\prod_{i=1}^{n} \prod_{j=1}^{n} \exp \left(\sum_{t=t_{m-1}+1}^{t_{m}} A_{i j, t} \cdot \log \theta_{m, z_{i m} z_{j m}}+\left(\sum_{t}^{n}\left(1-A_{i j, t}\right)\right) \cdot \log \left(1-\theta_{m, z_{i m} z_{j m}}\right)\right) \\
& =\prod_{i=1}^{n} \prod_{j=1}^{n} \exp \left(\sum_{t=t_{m-1}+1}^{t_{m}} A_{i j, t} \cdot \log \left(\frac{\theta_{m, z_{i m} z_{j m}}}{1-\theta_{m, z_{i m} z_{j m}}}\right)+d \cdot \log \left(1-\theta_{m, z_{i m} z_{j m}}\right)\right)
\end{aligned}
$$

By using the exponential family representation of the density the above equation can be written as

$$
\begin{aligned}
& \mathcal{L}\left(A_{m: m+1} \mid \mathbf{z}_{m}, \boldsymbol{\theta}_{k}\right) \propto \exp \left(\sum_{i=1}^{n} \sum_{j=1}^{n} \sum_{t=t_{m-1}+1}^{t_{m}} \boldsymbol{\tau}_{e}^{*}\left(A_{i j, t}\right) \cdot \boldsymbol{\eta}_{e}\left(\theta_{m, z_{i m} z_{j m}}\right)\right) \\
& \propto \exp \left(\sum_{i=1}^{n} \sum_{j=1}^{n} \boldsymbol{\tau}_{e}\left(\bar{A}_{i j, m}\right) \cdot \boldsymbol{\eta}_{e}\left(\theta_{m, z_{i m} z_{j m}}\right)\right)
\end{aligned}
$$

with $\boldsymbol{\tau}_{e}^{*}(x)=(x, 1), \boldsymbol{\tau}_{e}(x)=(x, d)$ and $\boldsymbol{\eta}_{e}(x)=(\log (x /(1-x)), \log (1-x))$.

Finally, the likelihood function of the change-point SBM is

$$
\begin{aligned}
& \mathcal{L}\left(A_{1: M} \mid \mathbf{z}_{1: M}, \boldsymbol{\theta}_{1: M}\right) \propto \prod_{m=1}^{M} \mathcal{L}\left(A_{m: m+1} \mid \mathbf{z}_{m}, \boldsymbol{\theta}_{m}\right) \\
& \propto \exp \left(\sum_{m=1}^{M} \sum_{i=1}^{n} \sum_{j=1}^{n} \boldsymbol{\tau}_{e}\left(\bar{A}_{i j, m}\right) \cdot \boldsymbol{\eta}_{e}\left(\theta_{m, z_{i m} z_{j m}}\right)\right)
\end{aligned}
$$

ii. Proof of the results in Eq. 11 


$$
\begin{aligned}
& \mathcal{L}\left(A_{m: m+1} \mid \mathbf{z}_{m}, \boldsymbol{\theta}_{k}\right)=\prod_{t=t_{m-1}+1}^{t_{m}} \prod_{(i, j) \in W_{m}} \mathcal{N}\left(A_{i j, t} \mid \mu_{m, z_{i m} z_{j m}}, \sigma_{m, z_{i m} z_{j m}}^{2}\right) \\
& =\prod_{i=1}^{n} \prod_{j=1}^{n} \exp \left(\sum_{t=t_{m-1}+1}^{t_{m}} A_{i j, t} \cdot \frac{\mu_{m, z_{i m} z_{j m}}}{\sigma_{m, z_{i m} z_{j m}}^{2}}-\sum_{t=t_{m-1}+1}^{t_{m}} A_{i j, t}^{2} \cdot \frac{1}{2 \sigma_{m, z_{i m} z_{j m}}^{2}}-d \cdot \frac{\mu_{m, z_{i m} z_{j m}}^{2}}{2 \sigma_{m, z_{i m} z_{j m}}^{2}}\right) \\
& \propto \exp \left(\sum_{i j} \boldsymbol{\tau}_{w}\left(\bar{A}_{i j}\right) \cdot \boldsymbol{\eta}_{w}\left(\boldsymbol{\theta}_{m, z_{i m} z_{j m}}\right)\right)
\end{aligned}
$$

where $\boldsymbol{\tau}_{w}(x)=\left(x, x^{2}, d\right)$ and $\boldsymbol{\eta}_{w}(\boldsymbol{\theta})=\left(\mu / \sigma^{2},-1 /\left(2 \sigma^{2}\right),-\mu^{2} /\left(2 \sigma^{2}\right)\right)$. Finally, the likelihood function of the change-point pure WSBM is

$$
\begin{aligned}
& \mathcal{L}\left(A_{1: M} \mid \mathbf{z}_{1: M}, \boldsymbol{\theta}_{1: M}\right) \propto \prod_{m=1}^{M} \mathcal{L}\left(A_{m: m+1} \mid \mathbf{z}_{m}, \boldsymbol{\theta}_{m}\right) \\
& \propto \exp \left(\sum_{m=1}^{M} \sum_{i=1}^{n} \sum_{j=1}^{n} \boldsymbol{\tau}_{w}\left(\bar{A}_{i j}\right) \cdot \boldsymbol{\eta}_{w}\left(\boldsymbol{\theta}_{m, z_{i m} z_{j m}}\right)\right)
\end{aligned}
$$

iii. Proof of the results in Eq. 12

Following the same line as in the proof of Eq. 9 and Eq. 11 we obtain

$$
\mathcal{L}\left(A_{m: m+1} \mid \mathbf{z}_{m}, \boldsymbol{\theta}_{k}\right) \propto \exp \left(\sum_{i j} \boldsymbol{\tau}_{e}\left(\bar{A}_{i j, m}\right) \cdot \boldsymbol{\eta}_{e}\left(\theta_{m, z_{i m} z_{j m}}\right)+\sum_{i j} \boldsymbol{\tau}_{w}\left(\bar{A}_{i j}\right) \cdot \boldsymbol{\eta}_{w}\left(\boldsymbol{\theta}_{m, z_{i m} z_{j m}}\right)\right)
$$

the likelihood function of the change-point WSBM is

$$
\begin{aligned}
& \mathcal{L}\left(A_{1: M} \mid \mathbf{z}_{1: M}, \boldsymbol{\theta}_{1: M}\right) \propto \prod_{m=1}^{M} \mathcal{L}\left(A_{m: m+1} \mid \mathbf{z}_{m}, \boldsymbol{\theta}_{m}\right) \\
& \propto \exp \left(\sum_{m=1}^{M} \sum_{i j} \boldsymbol{\tau}_{e}\left(\bar{A}_{i j, m}\right) \cdot \boldsymbol{\eta}_{e}\left(\theta_{m, z_{i m} z_{j m}}\right)+\sum_{m=1}^{M} \sum_{i j} \boldsymbol{\tau}_{w}\left(\bar{A}_{i j}\right) \cdot \boldsymbol{\eta}_{w}\left(\boldsymbol{\theta}_{m, z_{i m} z_{j m}}\right)\right)
\end{aligned}
$$




\section{B Patterns of Network connectivity}

In this Appendix, Figure 10 reports the type of potential connectivity patterns of the network communities distinguishing among intra and inter linkages. All of these types can be generated through the Weighted Stochastic Block model (Aicher et al., 2014).

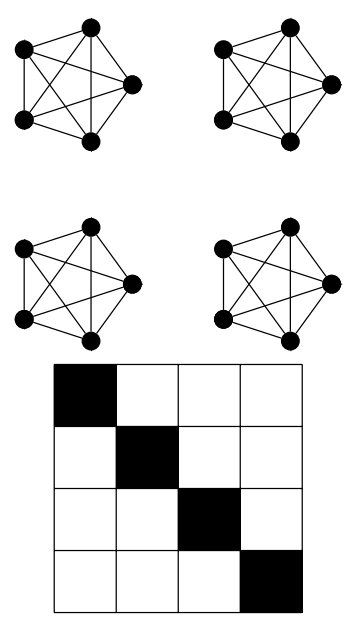

(a) Assortative

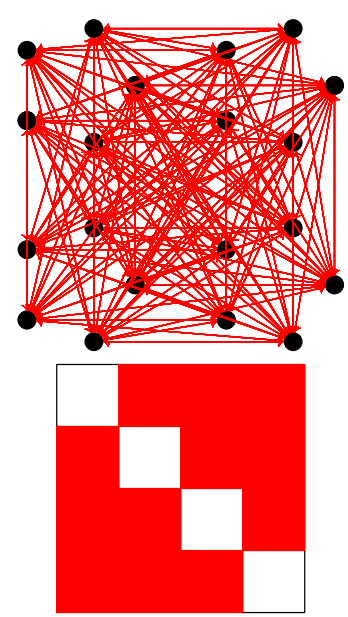

(b) Disassortative

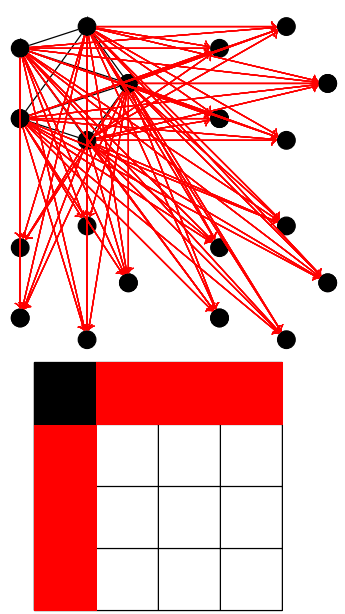

(c) Core-Periphery

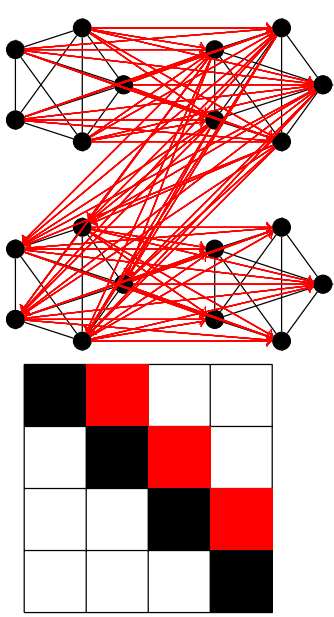

(d) Ordered

Figure 10: Examples of potential community connectivity patterns as in Aicher et al. (2014). The first (second) row reports the graph (adjacency matrix) of the network ordered by community membership of the nodes. Black (red) colour indicates intra (inter) linkages. (a) Assortative type shows mainly intra-linkages within community. (b) Disassortative type shows inter-linkages in between communities. (c) Core-Periphery type shows the presence of a core with intra-linkages and inter-linkages to the periphery (the other communities) which is in turn mainly connected to the core. and (d) Ordered type shows inter-linkages and intra-linkages from the top to the bottom. 


\section{Optimal Number of Block and Model Selection}

In this section, we consider three settings of the WSBM: i) SBM with $\alpha=1$, balanced WSBM with $\alpha=0.5$ and pure WSBM with $\alpha=0$. First, we select the optimal number of blocks for each window obtained with the three considered models and then comparing the community structures across the models. Then, we perform the model selection according to their predictive ability through the tuning parameter $\alpha$ and we discuss the optimal block numbers as an indicator of systemic risk.

\section{C.1 Optimal Number of Blocks}

We select the optimal number of blocks through the marginal log-likelihoods approximated by the lower bound, $G(q)$, of each model and then, we compare them by varying the number of $K$ communities from 1 to the number of total nodes in the network $n$. The optimal number

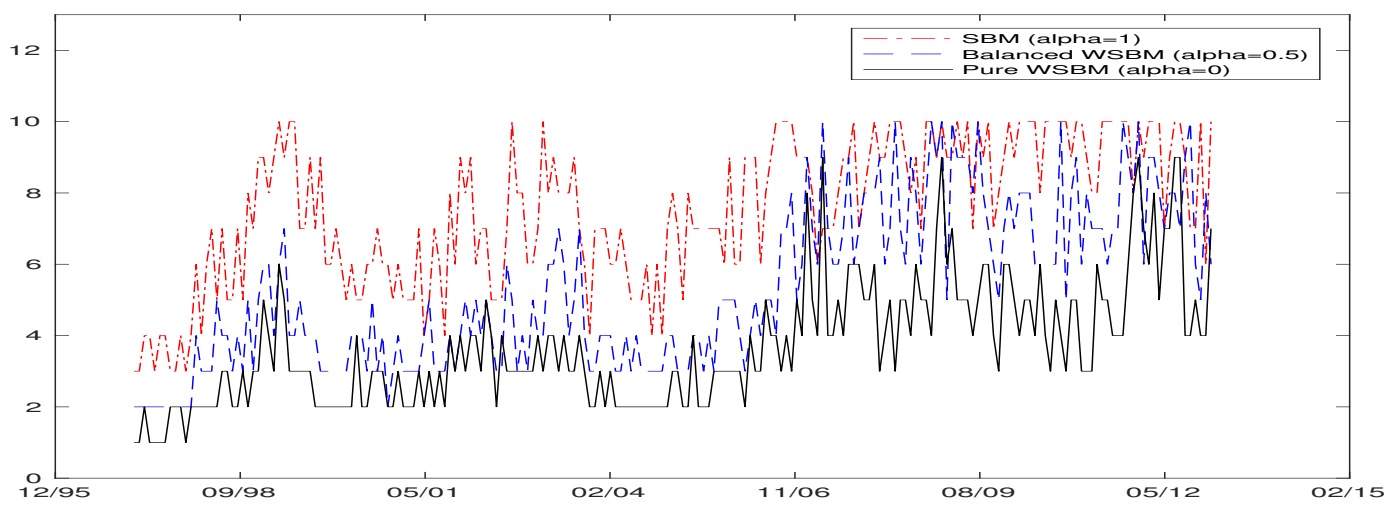

Figure 11: Optimal number of latent blocks, $K$, over time: black dash-dot line $(\alpha=1)$, red solid line and blue dashed line represent $\alpha=0.5$ and $\alpha=0$ respectively.

of latent blocks over time with three different values of tuning parameter $\alpha$ are reported in the Figure 11 At the first glance, it is clearly visible that the the optimal number of blocks of all the three considered models are increasing in the crisis periods. In fact, during the periods of 2007-2008 global crisis and European sovereign debt crisis, the optimal block numbers reach their highest levels. Clearly, the optimal block numbers considerably differs from the model to model. Most of the time, the values of the optimal block numbers with $\alpha=1$ and $\alpha=0$ act as an upper and lower bound for the model $\alpha=0.5$, respectively. As reported in Table 7, the optimal number of blocks reaches the highest level (10) in 50 windows for the $\operatorname{SBM}(\alpha=1)$ model while the balanced WSBM $(\alpha=0.5)$ reaches this level only in 13 windows. In the pure WSBM $(\alpha=0)$, the highest number of optimal block is 7 .

We proceed with the selection of the model by comparing the SBM $(\alpha=1)$, the balanced WSBM 


\begin{tabular}{|c|c|l|}
\hline \multicolumn{3}{|c|}{ With $\alpha=1$} \\
\hline K & Windows & Percent \\
\hline 2 & 11 & $5.26 \%$ \\
3 & 7 & $3.35 \%$ \\
4 & 47 & $22.49 \%$ \\
5 & 37 & $17.70 \%$ \\
6 & 18 & $8.61 \%$ \\
7 & 3 & $1.44 \%$ \\
8 & 14 & $6.70 \%$ \\
9 & 22 & $10.53 \%$ \\
10 & 50 & $23.92 \%$ \\
\hline
\end{tabular}

\begin{tabular}{|c|c|l|}
\hline \multicolumn{3}{|c|}{ With $\alpha=0.5$} \\
\hline K & Windows & Percent \\
\hline 2 & 15 & $7.18 \%$ \\
3 & 28 & $13.40 \%$ \\
4 & 51 & $24.40 \%$ \\
5 & 25 & $11.96 \%$ \\
6 & 22 & $10.53 \%$ \\
7 & 15 & $7.18 \%$ \\
8 & 22 & $10.53 \%$ \\
9 & 18 & $8.61 \%$ \\
10 & 13 & $6.22 \%$ \\
\hline
\end{tabular}

\begin{tabular}{|c|c|c|}
\hline \multicolumn{3}{|c|}{ With $\alpha=0$} \\
\hline $\mathrm{K}$ & Windows & Percent \\
\hline 2 & 53 & $25.36 \%$ \\
3 & 60 & $28.71 \%$ \\
4 & 31 & $14.83 \%$ \\
5 & 22 & $10.53 \%$ \\
6 & 27 & $12.92 \%$ \\
7 & 10 & $4.78 \%$ \\
8 & 6 & $2.87 \%$ \\
9 & 0 & $0.00 \%$ \\
10 & 0 & $0.00 \%$ \\
\hline
\end{tabular}

Table 7: The optimal block numbers of the models, SBM $(\alpha=1)$, balanced WSBM $(\alpha=0.5)$ and pure WSBM $(\alpha=0)$.

( $\alpha=0.5)$ and the pure WSBM $(\alpha=0)$ and then we discuss the selection of tuning parameter, $\alpha$. In order to make a viable comparison and show the structural difference of the communities among the considered models, we fix the number of blocks equal to $K=4$. The $18^{\text {th }}$ network obtained from SBM $(\alpha=1)$, Balanced WSBM $(\alpha=0.5)$ and Pure WSBM $(\alpha=0)$ are shown in Figure 12, In the SBM $(\alpha=1)$, Figure 12(a)-(b), the most systematically important community is block 3 in which the members have the highest out degree. However, there are not particular edge weight characteristics in SBM $(\alpha=1)$ communities. In the balanced WSBM $(\alpha=0.5)$ the most systematically important community is block 2. Not surprisingly, the standard deviation of the edge weights is rather lower than SBM $(\alpha=1)$ than the ones of its communities since the model learns from both edge existence and edge weight information. In pure WSBM $(\alpha=0)$, however, the blocks are rather homogeneous in terms of in and out degree as in Figure 12(c)-(d)) which is quite expected since pure WSBM $(\alpha=0)$ learns only from the edge weight information. Therefore, except the pure WSBM, each block has different connectivity characteristics in SBM and balanced WSBM networks. On the other hand, each block has different edge weight level characteristics in pure WSBM (see Figure 12(c)-(d)).

\section{C.2 Model Selection and $\alpha$-Calibration}

The selection of tuning parameter $(\alpha)$ represents a crucial step in terms of modeling decision. Clearly, the ideal choice should prefer the model which can exploit all the available information such as connectedness and edge weights information. Thus, we compare the performances of the models in terms of edge and edge weight prediction performances to show the ability of each model. The combined forecast method introduced in Bates and Granger (1969) is applied by giving equal weights to each of individual forecasts of SBM $(\alpha=1)$, WSBM $(\alpha=0.5)$ and pure

\footnotetext{
${ }^{7}$ The last date of the $18^{\text {th }}$ window is 27 May 1998
} 


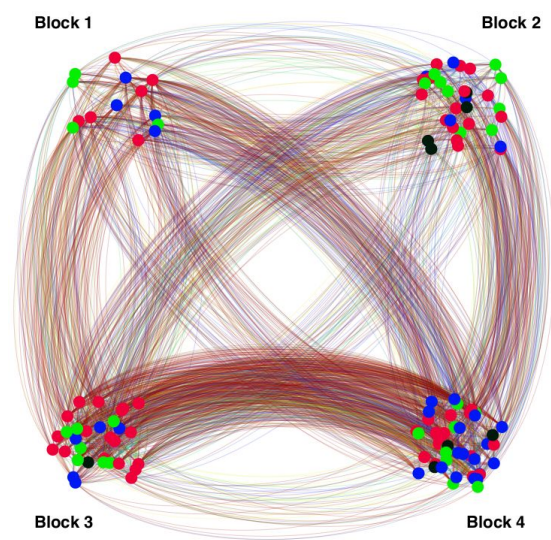

(a) $\operatorname{SBM}(\alpha=1)$

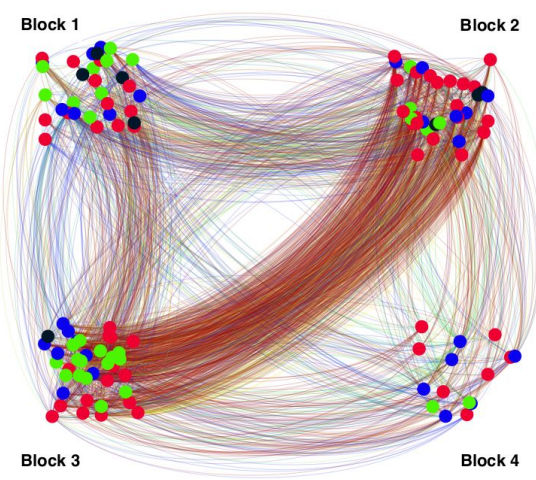

(c) Balanced WSBM $(\alpha=0.5)$

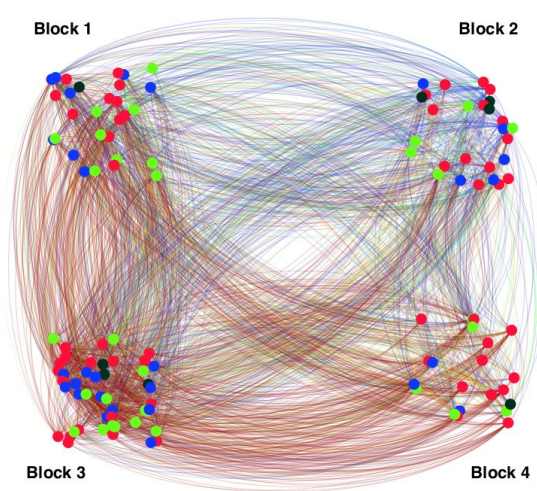

(e) Pure WSBM $(\alpha=0)$

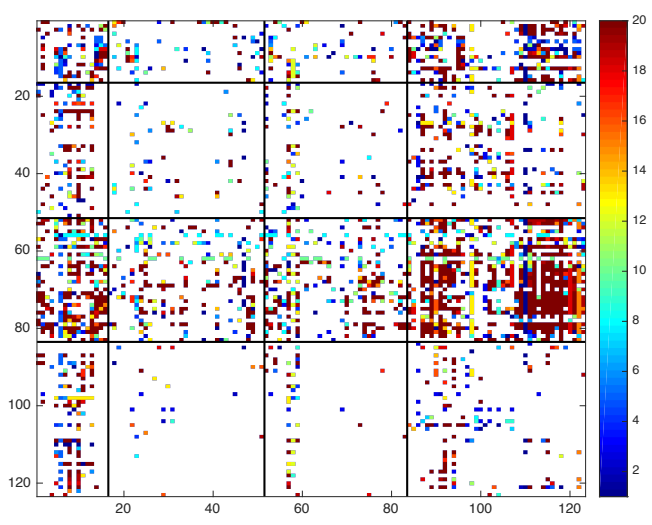

(b) SBM Adjacency Matrix

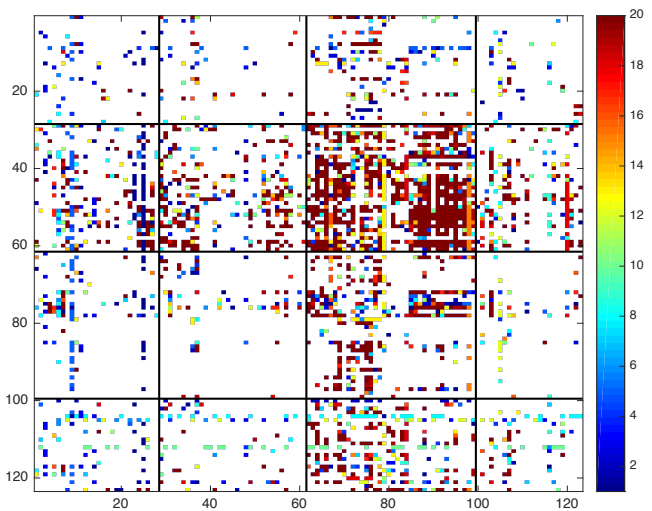

(d) Balanced WSBM Adjacency Matrix

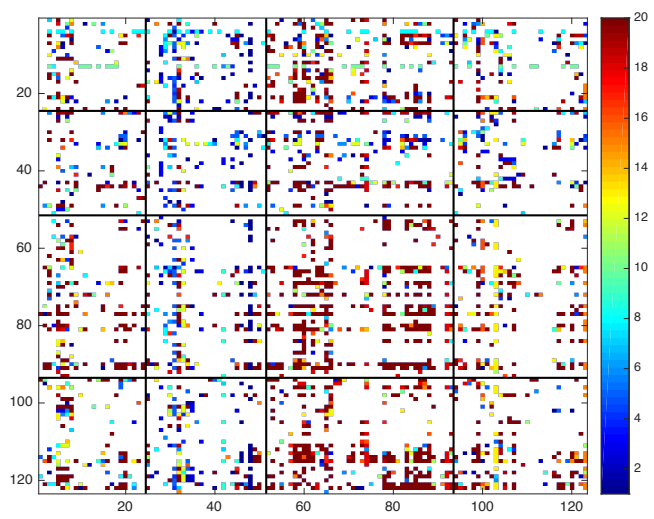

(f) Pure WSBM Adjacency Matrix

Figure 12: European Financial Institution Network $18^{\text {th }}$ Window, 27 May 1998. Red nodes are banks, blue nodes are insurance companies, green nodes are companies in Financial Service sector, black nodes are Real Estate Companies. In the Nodal Networks, block 1, and 2 are in the first row, block 3 and 4 are in the second row. 
WSBM $(\alpha=0)]^{8}$ To show the contribution of each model's forecast performance out of the combined forecast for each window, the following calculation is done:

$$
W_{i t}=\frac{M S E_{i t}^{-1}}{\sum_{i=1}^{3} M S E_{i t}^{-1}}, \quad i=1,2,3 \quad \text { and } \quad t=1,2, \ldots, 209
$$

where $t$ represents the number of the window, $t^{t h}$ window, MSE indicates average mean square error, forecast error, and $i$ is the model typ $\AA^{9}, W_{i t}$ indicates the relative forecast performance of model $i$ at the $t^{t h}$ window. Therefore, by Equation 28, $W_{i t}>W_{-i t}$ indicates the best forecast performance at window $t$ is model.

The relative forecast performances of all the models, $\operatorname{SBM}(\alpha=1)$, balanced WSBM $(\alpha=0.5)$ and pure WSBM $(\alpha=0)$, are presented in Figure 13 by combined forecast method. Clearly, the balanced WSBM $(\alpha=0.5)$ performs better on edge and edge weight prediction tasks (see Figure 13(a)). In addition, to analyze the features of the models in detail, the models' performances on edge existence and edge weigh predictions are provided separately (see Figure 13(b)-(c)).

As shown in Figure 13(b), the SBM $(\alpha=1)$ and balanced WSBM $(\alpha=0.5)$ are the most accurate models most of the time with a high margin. In addition, $\operatorname{SBM}(\alpha=1)$ predicts edge existences better than balanced WSBM $(\alpha=1)$ which is quite expected because $\operatorname{SBM}(\alpha=1)$ is solely sensitive to edge existences information. However, the balanced WSBM $(\alpha=0.5)$ considers both edge existence and weight information and it also performs well on the same task as $\operatorname{SBM}(\alpha=1)$. Not surprisingly, the poorest model on edge prediction task is the pure WSBM $(\alpha=0)$. On the edge weights prediction task, however, the pure WSBM $(\alpha=0)$ is the most accurate, often by a large margin and it is not unexpected as well, because it is modeled to learn only from edge weight information (see Figure 13(c)). The balanced WSBM $(\alpha=0.5)$ also performs quite accurate on the edge weight prediction task as nearly as the pure WSBM $(\alpha=0)$. The SBM $(\alpha=1)$, however, is the worst model on edge weight prediction task which is quite expected by construction.

In general, the SBM $(\alpha=1)$ is the best model for the performance of edge prediction but very poor on edge weight predictions. The pure WSBM $(\alpha=0)$ performs accurate on weight prediction but it is very poor on edge prediction. However, the balanced WSBM $(\alpha=0.5)$ is the only model which performs well on both tasks (see Figure 13). It performs as well as the SBM

\footnotetext{
${ }^{8}$ For sake of clarity, we include for each network window, the average mean square errors on edge existence and edge weight predictions of SBM $(\alpha=1)$, balanced WSBM $(\alpha=0.5)$ and pure WSBM $(\alpha=0)$ with $90 \%$ confidence interval in Supplementary material.

${ }^{9}$ Where $i=1$ denotes SBM $(\alpha=1), i=2$ balanced WSBM $(\alpha=0.5)$ and $i=3$ pure WSBM $(\alpha=0)$
} 

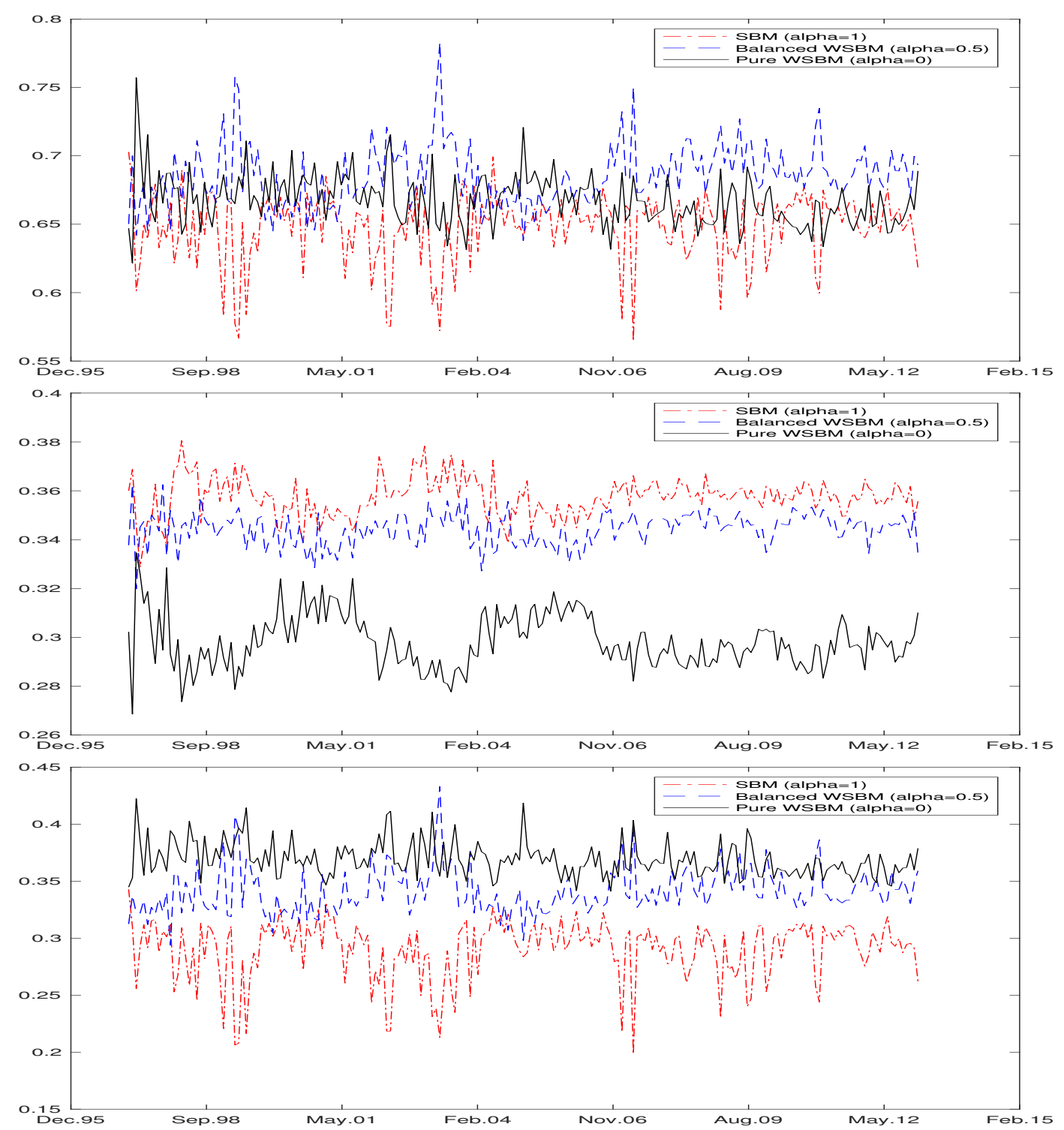

Figure 13: Edge existence and weight predictions performances of the models: a) Edge existence and edge weight prediction performances of the models (Top panel); b) Performances of the models only on edge existence predictions (Mid panel) and c) Performances of the models only on edge weight predictions (Bottom panel).

in edge prediction and substantially better than the SBM in edge weight prediction. In other words, the balanced WSBM $(\alpha=0.5)$ is more powerful model either than the $\operatorname{SBM}(\alpha=1)$ and pure WSBM $(\alpha=0)$ in terms of prediction performances. 


\section{Robustness Checks}

As robustness check, we estimate the optimal block numbers with different window lengths of 5 , 10, 15, 20, 25 and 30 business days. As shown in Figure 14, the dynamic of the optimal number of $K$ is not affected and remains stable with different window lengths.
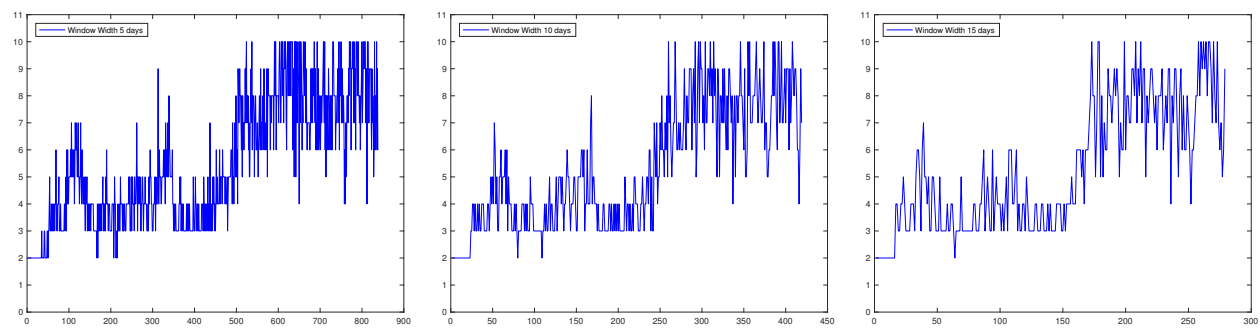

(a) Optimal block numbers of (b) Optimal block numbers of (c) Optimal block numbers of Balanced WSBM with win- Balanced WSBM with win- Balanced WSBM with window widths of 5 business dow widths of 10 business dow widths of 15 business days. days. days.
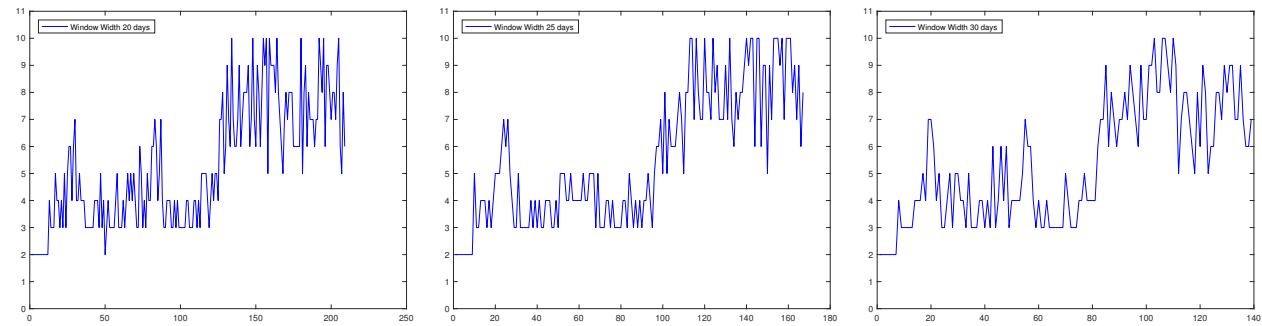

(d) Optimal block numbers of (e) Optimal block numbers of (f) Optimal block numbers of Balanced WSBM with win- Balanced WSBM with win- Balanced WSBM with window widths of 20 business dow widths of 25 business dow widths of 30 business days. days. days.

Figure 14: Robustness of the optimal number of blocks with different non-overlapping window widths 


\section{E Additional Connectivity Measures}

In this section, we report in Figure 15 the additional connectivity measures such as in(out), in(out)-inter and in(out)-intra degrees.

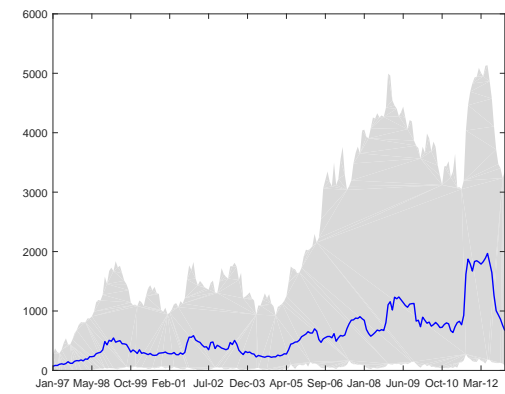

(a) Total in degrees.

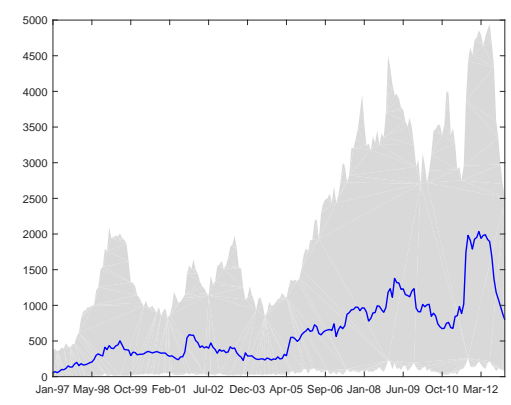

(d) Total out degrees.

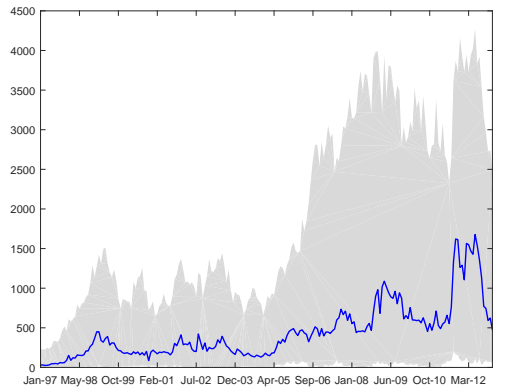

(b) In-inter degrees.

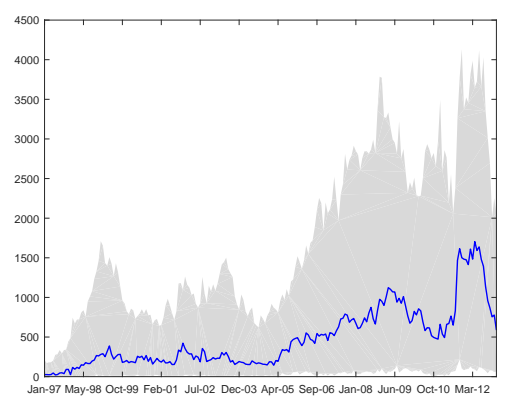

(e) Out-inter degrees.

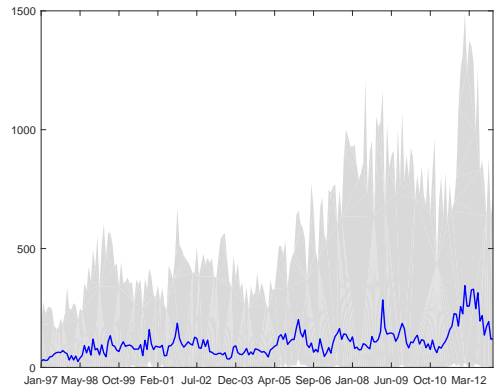

(c) In-intra degrees.

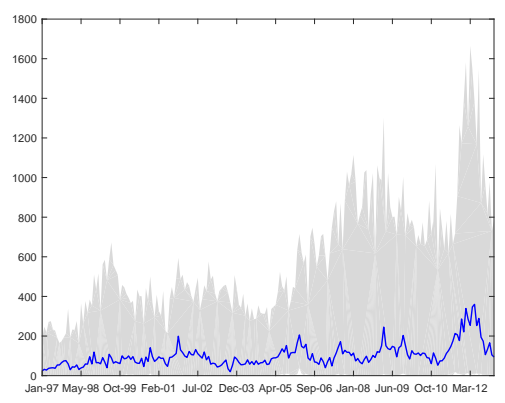

(f) Out-intra degrees.

Figure 15: $95 \%$ high density region (gray area) and the cross-section mean (solid blue line) of total in(out) degrees (first/forth panel), in(out)-inter degrees (second/fifth panel) and in(out)intra degrees (third/sixth panel) for the European financial network over time. 


\section{F Early warning indicator using eigenvector centrality}
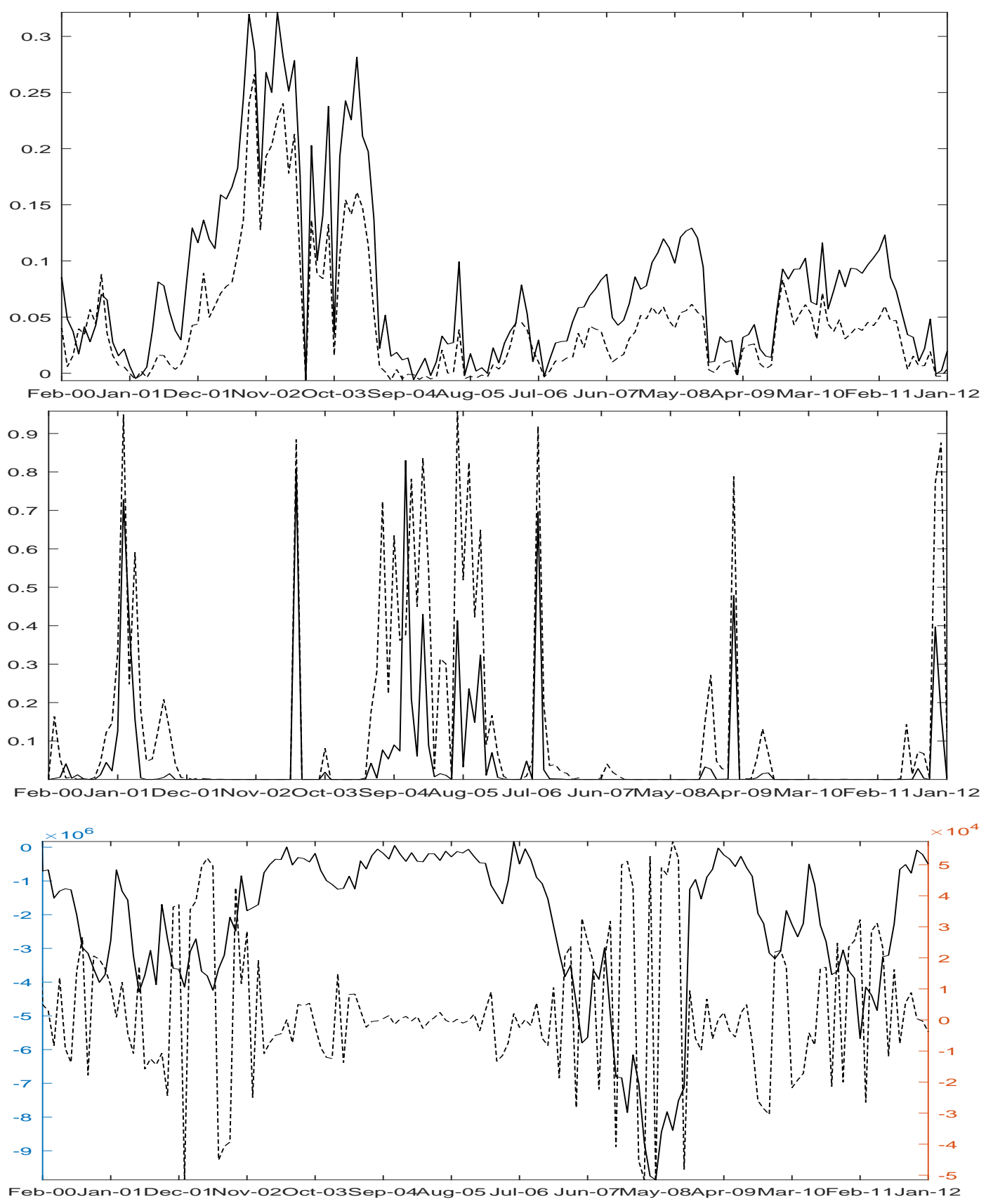

Figure 16: Adjusted R-squared (top), coefficient p-value (mid) and coefficient magnitude (bottom) for the cross-sectional regressions where the dependent variable is the maximum loss incurred by a single institution within the next two years and the independent variable is alternatively the out-inter community degrees (solid line) and the eigenvector centrality (dashed line). See Equation 5.1. Note: The coefficient magnitude are reported on the left y-axis for the out-inter community degrees and on the right y-axis for the eigenvector centrality. 


\section{Recent Issues}

No. 207 Claes Bäckman, Tobin Hanspal

No. 206 Loriana Pelizzon, Anjan Thakor, Calebe de Roure

No. 205 Horst Entorf, Jia Hou

No. 204 Loriana Pelizzon, Matteo Sottocornola

No. 203 Florian Hett, Felix Schmidt

No. 202 Tobias H. Tröger

No. 201 Dirk Krueger, Alexander Ludwig

No. 200 Nils Grevenbrock, Max Groneck, Alexander Ludwig, Alexander Zimper

No. 199 Tobias H. Tröger

No. 198 Henning Hesse, Boris Hofmann, James Weber

No. 197 Benjamin Clapham, Peter Gomber, Martin Haferkorn, Paul Jentsch, Sven Panz

No. 196 Benjamin Clapham, Peter Gomber, Sven Panz

No. 195 Benjamin Clapham, Peter Gomber, Martin Haferkorn, Sven Panz

No. 194 Baptiste Massenot

No. 193 Mario Bellia, Roberto Panzica, Loriana Pelizzon, Tuomas Peltonen
The Geography of Alternative Work

P2P Lending versus Banks: Cream Skimming or Bottom Fishing?

Financial Education for the Disadvantaged? A Review

The Impact of Monetary Policy Interventions on the Insurance Industry

Pushing Through or Slacking Off? Heterogeneity in the Reaction to Rank Feedback

Germany's Reluctance to Regulate Related Party Transactions

Optimal Taxes in the OLG Model with Uninsurable Idiosyncratic Income Risk

Cognition, Optimism and the Formation of Age-Dependent Survival Beliefs

Regulation of Crowdfunding in Germany

The Macroeconomic Effect of Asset Purchases Revisited

Circuit Breakers - A Survey among International Trading Venues

Coordination of Circuit Breakers? Volume Migration and Volatility Spillover in Fragmented Markets

Managing Excess Volatility: Design and Effectiveness of Circuit Breakers

A Business Cycle Model with Neuroeconomic Foundations

The Demand for Central Clearing: To Clear or Not to Clear, That is the Question 